\title{
Quantum volume and length fluctuations in a midi-superspace model of Minkowski space
}

\author{
Jeremy Adelman, ${ }^{1}$ Franz Hinterleitner, ${ }^{2}$ and Seth Major ${ }^{3}$ \\ ${ }^{1}$ Department of Physics, University of California, \\ One Shields Avenue, Davis CA 95616 USA \\ ${ }^{2}$ Department of Theoretical Physics and Astrophysics, \\ Faculty of Science of the Masaryk University, \\ Kotlárská 2, 61137 Brno, Czech Republic \\ ${ }^{3}$ Department of Physics, Hamilton College, Clinton NY 13323 USA
}

(Dated: January 2015)

\begin{abstract}
In a (1+1)-dimensional midi-superspace model for gravitational plane waves, a flat space-time condition is imposed with constraints derived from null Killing vectors. Solutions to a straightforward regularization of these constraints have diverging length and volume expectation values. Physically acceptable solutions in the kinematic Hilbert space are obtained from the original constraint by multiplying with a power of the volume operator and by a similar modification of the Hamiltonian constraint, which is used in a regularization of the constraints. The solutions of the modified Killing constraint have finite expectation values of geometric quantities. Further, the expectation value of the original Killing constraint vanishes, but its moment is non-vanishing. As the power of the volume grows the moment of the original constraint grows, while the moments of volume and length both decrease. Thus, these states provide possible kinematic states for flat space, with fluctuations. As a consequence of the regularization of operators the quantum uncertainty relations between geometric quantities such as length and its conjugate momentum do not reflect naive expectations from the classical Poisson bracket relations.
\end{abstract}

PACS 04.20.Fy, 04.30.Nk, 04.60.Ds, 04.60.Pp 


\section{INTRODUCTION}

Loop quantum gravity (LQG) quantizes the spatial geometry by introducing "atoms of spatial geometry" in form of quanta of volume, area, length, and angle [1 6]. Unlike the Minkowski vacuum in quantum field theories of different kinds of matter, the quantum model of flat space appears to be, not a "no particle" state, but rather a highly excited state with a macroscopically homogeneous distribution of excited quanta of geometry. In this paper we explore the nature of quantum flat space in an effectively $(1+1)$-dimensional gravitational system tailored to study the propagation of plane gravitational waves. This is the third paper in a series [7, 8] on the quantization of gravitational plane waves with the eventual goal of quantizing "small amplitude" plane gravitational waves on flat space and to ascertain the effects (if any) of the underlying fundamental geometric discreteness of LQG on the propagation of waves. This work, identifying candidate kinematic states of flat space, is a step toward that goal.

One advantage of the present approach is that we can derive model states from classical flatness conditions, in the form of constraints on quantum states derived from the existence of Killing vector fields. Additionally, the resulting algebra of constraints, including these new "Killing constraints", is first class [8]. The second, main advantage of the present - so far kinematical - model is the realistic chance to subject them to quantum dynamics. For, even if the midi-superspace Hamiltonian constraint is not simple, it is not so complicated that, from the very beginning, it thwarts the application of the dynamics to the candidate flat space states described here.

One important aspect of the problem that this paper does not address is the derivation of physical states. Although the present quantization uses the Hamiltonian constraint in formulating quantum kinematic constraints, we do not obtain physical solutions or check that the constraint algebra is anomaly-free. Work on this is underway.

The plane wave class of pp-wave space-times considered here are derived from the cosmological Gowdy model, which was quantized by Banerjee and Date [9] using formal tools from earlier work by Bojowald and Swiderski [10]. Our earlier work on plane gravitational waves [8], shows that left- or right-moving wave space-times can be found using a system of first-class constraints. In this approach a description of background flat space for wave propagation arises in a natural way. 
Earlier work addressed similar models of gravitational waves. Neville considered the quantization of plane gravitational waves both within geometrodynamics and with complex connections [11]. Borissov studied plane waves and weave states [12]. To quantize a similar model Beetle exploited the observation that the symmetry reduction of non-compact toroidally symmetric space-times yields a system equivalent to a free massless scalar field on a fixed $(2+1)$-dimensional background [13]. Using metric variables Mena Marugán and Montejo reduced the model at the classical level using gauge choices and symmetry reduction [14]. These quantizations leave the relation between the fundamental discrete geometry of LQG and classical local Lorentz invariance veiled. Since this relation is precisely what we wish to elucidate, we take an approach to quantization closer to that of $(3+1)$-dimensional LQG.

The organization of the paper is the following: In the next section and Section IV we briefly present those basics of the quantized Gowdy model that are necessary for our adaptation to gravitational waves - opening up the global toroidal topology to flat space and finding a set of first-class "Killing constraints" that select unidirectional gravitational waves [7, 8]. The geometric quantities used in the analysis of the flat space constraints are defined in Section III. In this section we also interpret the constraints geometrically in terms of the rate of change of cross section areas and in terms of length. We show in IVC that a set of simple "vanishing curvature" constraints yields non-normalizable states in the kinematic Hilbert space, further motivating the use of the constraints derived from the Killing vectors.

We select quantum states for flat geometry by imposing a "no wave" constraint derived from the left- and right-moving constraints of Ref. [8]. These Killing constraints, formulated and implemented in Sections $\mathrm{V}$ and $\mathrm{VI}$, suppress all waves and thus give "no-wave states", a kinematic model for flat space. Calculating expectation values and fluctuations in geometric quantities around these solutions, we find that requiring finite expectation values of geometric quantities limits the formulation of the "no wave" constraint.

The main work of this paper is dedicated to the construction and analysis of the candidate flat space states, as detailed in Sections VI and VII, as well as the appendices. The constraint operators contain explicit connection components, which cannot be directly promoted to quantum operators, and must be regularized. We present two different strategies to do this, one by applying "Thiemann's trick" [15] to replace the constraint by the commutator of a part of the Hamiltonian constraint with the volume operator, and the other one by 
directly approximating the connection in terms of corresponding holonomies and obtaining a Hermitian constraint. In both cases it turns out that the "no-wave constraints" in their straightforward form are too strong - they produce states with diverging length and volume expectation values, even for a single spin network node or vertex. We call this smallest possible unit an "atom of geometry".

We consider two different ways of relaxing the no-wave constraints using the volume operator. These have normalizable solutions with finite expectation values and uncertainties for geometric quantities on a single atom of geometry, giving candidate states for flat space as subset of the kinematical state space of a single atom of geometry. The kinematical Hilbert space of the whole system is subtile due to the open topology. (See the work by Thiemann and Winkler [16]). We have not addressed the normalizability of the constraints in that setting.

\section{VARIABLES AND CONSTRAINTS}

The space-time model for plane gravitational waves propagating in the $z$-direction, where the $x$ and the $y$ direction form a plane of homogeneity, is formally very close to the polarized Gowdy model in Ref. [9]. The difference lies only in the global topology; locally both models are formulated in the same Ashtekar-type variables. Following Ref. [9], we introduce densitized triads in a space-like hypersurface with the component $\mathcal{E}(z)$ in the inhomogeneous $z$-direction and the homogeneous transverse components arranged as two-vectors

$$
\vec{E}^{x}=\left(E^{x} \cos \eta, E^{x} \sin \eta\right), \quad \vec{E}^{y}=\left(-E^{y} \sin \eta, E^{y} \cos \eta\right)
$$

We consider only polarized gravitational waves, where these two vectors are orthogonal. Like $\mathcal{E}$, the components $E^{x}, E^{y}$, and $\eta$ are functions of $z$. In terms of these variables the spatial metric is given by

$$
\mathrm{d} s^{2}=\mathcal{E} \frac{E^{y}}{E^{x}} \mathrm{~d} x^{2}+\mathcal{E} \frac{E^{x}}{E^{y}} \mathrm{~d} y^{2}+\frac{E^{x} E^{y}}{\mathcal{E}} \mathrm{d} z^{2} .
$$

The canonically conjugate variable to $\mathcal{E}$ is the Ashtekar-Barbero connection $\mathcal{A}(z)$, conjugate variables to $E^{x}$ and $E^{y}$ are $X(z)$, and $Y(z)$, the extrinsic curvature components $K_{x}$ and $K_{y}$, rescaled by multiplication with the Barbero-Immirzi parameter $\gamma$. The angular variable $\eta$ represents a pure gauge degree of freedom. Its conjugate momentum $P_{\eta}(z)$ is the 
generator of $U(1)$ rotations in the $(x, y)$ plane. The non-vanishing Poisson brackets are

$$
\begin{aligned}
& \left\{\mathcal{A}(z), \mathcal{E}\left(z^{\prime}\right)\right\}=\left\{\eta(z), P_{\eta}\left(z^{\prime}\right)\right\}=\left\{X(z), E^{x}\left(z^{\prime}\right)\right\}=\left\{Y(z), E^{y}\left(z^{\prime}\right)\right\} \\
& =\kappa \gamma \delta\left(z-z^{\prime}\right)
\end{aligned}
$$

where $\kappa$ is proportional to Newton's constant [24]. Given the symmetries of the model the standard gauge-generating constraints reduce to the Gauß constraint

$$
G=\frac{1}{\kappa \gamma}\left(\mathcal{E}^{\prime}+P_{\eta}\right)
$$

the diffeomorphism constraint

$$
C=\frac{1}{\kappa \gamma}\left[X^{\prime} E^{x}+Y^{\prime} E^{y}-\mathcal{E}^{\prime} \mathcal{A}+\eta^{\prime} P_{\eta}\right]
$$

and the Hamiltonian constraint,

$$
\begin{aligned}
& H=-\frac{1}{\kappa \sqrt{\mathcal{E} E^{x} E^{y}}}\left[\frac{1}{\gamma^{2}}\left\{X E^{x} Y E^{y}+\mathcal{E}\left(\mathcal{A}+\eta^{\prime}\right)\left(X E^{x}+Y E^{y}\right)\right\}+\frac{1}{4}\left(\mathcal{E}^{\prime}\right)^{2}+\right. \\
& \left.\frac{1}{4} \mathcal{E}^{2}\left(\frac{\left(E^{x}\right)^{\prime}}{E^{x}}-\frac{\left(E^{y}\right)^{\prime}}{E^{y}}\right)^{2}\right]+\frac{1}{\kappa}\left(\frac{\mathcal{E} \mathcal{E}^{\prime}}{\sqrt{\mathcal{E} E^{x} E^{y}}}\right)^{\prime}-\frac{\kappa G^{2}}{4 \sqrt{\mathcal{E} E^{x} E^{y}}}-\gamma\left(\sqrt{\frac{\mathcal{E}}{E^{x} E^{y}}} G\right)^{\prime} .
\end{aligned}
$$

A prime denotes the derivative with respect to $z$. As the last two terms of $H$ contain the Gauß constraint, they may be dropped when the constraint is applied to gauge-invariant states [25]. When implemented these constraints are integrated with test functions. For instance, the Hamiltonian constraint is integrated with the lapse and denoted $H[N]=\int d z N H$, as usual. With four canonical pairs of field variables and three first-class constraints the system has one physical degree of freedom, which is realized by polarized waves, moving in either direction along the $z$-axis.

We use two parts of the Hamiltonian constraint in subsequent sections. The evolution of geometric quantities defined in the next section requires the kinetic part $H_{K}$ of $H$, which contains the conjugate variables $\mathcal{A}, X$, and $Y$, and is defined as in Ref. [9] by the decomposition of the Hamiltonian constraint

$$
H=-\frac{1}{\kappa}\left(H_{K}+H_{P}\right)
$$

with

$$
H_{K}[N]=\frac{1}{\gamma^{2}} \int \mathrm{d} z N(z) \frac{X E^{x} Y E^{y}+\mathcal{E}\left(\mathcal{A}+\eta^{\prime}\right)\left(X E^{x}+Y E^{y}\right)}{\sqrt{\mathcal{E} E^{x} E^{y}}}(z)
$$


To construct the "no wave" constraint we use the first term of $H_{K}$,

$$
H_{K}^{1}[N]=\frac{1}{\gamma^{2}} \int \mathrm{d} z N(z) \frac{X E^{x} Y E^{y}}{\sqrt{\mathcal{E} E^{x} E^{y}}} .
$$

Classically, we know that colliding waves produce a singularity [17]. For this reason, and the fact that the goal is to investigate loop quantization and the dispersion of gravitational waves, we further reduce the system to waves propagating in only one direction [8]. A spacetime with gravitational waves propagating exclusively in the negative $z$-direction has a null Killing vector field in this direction, related to uniform wave front propagation at the speed of light. As shown in Ref. [8], the existence of this Killing vector field yields a first-class "left-moving constraint"

$$
U_{+}:=X E^{x}+Y E^{y}+\gamma \mathcal{E}^{\prime}=0 .
$$

Analogously, the first-class constraint

$$
U_{-}:=X E^{x}+Y E^{y}-\gamma \mathcal{E}^{\prime}=0
$$

restricts to waves in the positive $z$ direction. Note that in Ref. [8] $U_{-}$is defined as a different linear combination of constraints that doesn't have the right-moving interpretation. The old $U_{-}$of Ref. [8] does not form a first-class algebra with the Hamiltonian and diffeomorphism constraints, whereas the current definition does. The Poisson brackets of the smeared-out constraint $U_{+}[f]=\int \mathrm{d} z f(z) U_{+}(z)$ with test function $f$ and the other constraints are weakly equal to zero.

$$
\begin{aligned}
& \left\{U_{+}[f], G[g]\right\}=0, \quad\left\{U_{+}[f], C[g]\right\}=-U_{+}\left[f^{\prime} g\right] \approx 0, \\
& \left\{U_{+}[f], H[N]\right\}=-U_{+}\left[\sqrt{\frac{\mathcal{E}}{E^{x} E^{y}}} f^{\prime} N\right]-\kappa \gamma H[f N] \approx 0 .
\end{aligned}
$$

Equation (13) contains the non-trivial structure function $\sqrt{\frac{\mathcal{E}}{E^{x} E^{y}}}=\sqrt{g^{z z}}$, the square root of the inverse metric component in the $z$-direction. Upon quantization, this structure function becomes operator-valued and this may lead to a quantum anomaly: The Dirac quantization procedure of determining physical states by the condition that they be annihilated by the constraint operators can be consistently carried out with equation (13), when $U_{+}$stands to the right of the structure function. Otherwise new constraints may arise. For the full theory it is shown in Ref. [19] that a well-defined Hamiltonian constraint is constructed from an operator ordering such that the connection variables are left of the triad variables. For the 
spherically symmetric case, similar arguments are given in Ref. [10]. With such a factor ordering (or, also in symmetric ordering) the first-class Poisson bracket relation (13) does not obviously carry over to quantum theory without modification.

Before constructing plane-wave solutions, we construct flat space solutions as backgrounds for wave propagation. Flat space is modeled as a state without left- as well as right-moving waves. By imposing both left- and right-moving constraints we suppress all waves and arrive at a $(1+1)$-dimensional model without waves. Classically, imposing $U_{+}$and $U_{-}$ simultaneously means

$$
\mathcal{K}:=X E^{x}+Y E^{y}=0
$$

and

$$
\mathcal{E}^{\prime}=0
$$

These constraints, especially the first, will be the focus of the rest of this work. Together, the constraints form a first-class algebra with the constraints of general relativity: They commute with the Gauß constraint. The Poisson brackets with the Hamiltonian constraint are

$$
\left\{\mathcal{E}^{\prime}[f], H[N]\right\}=-\frac{1}{\gamma} \mathcal{K}\left[\sqrt{\frac{\mathcal{E}}{E^{x} E^{y}}} f^{\prime} N\right], \quad\{\mathcal{K}[f], H[N]\}=-\gamma \mathcal{E}^{\prime}\left[\sqrt{\frac{\mathcal{E}}{E^{x} E^{y}}} f^{\prime} N\right]-\kappa \gamma H[f N],
$$

With the diffeomorphism constraint the brackets are

$$
\{\mathcal{K}[f], C[g]\}=-\mathcal{K}\left[f^{\prime} g\right], \quad\left\{\mathcal{E}^{\prime}[f], C[g]\right\}=-\mathcal{E}^{\prime}\left[f^{\prime} g\right]
$$

Arising from the existence of a Killing vector field, we call these pair of constraints the "Killing constraints". (The singular will refer to the first constraint, $\mathcal{K}=0$.) The second constraint obviously expresses homogeneity in the $z$-direction. The first constraint expresses homogeneity in the time direction, as we will see in the next section. Additionally, the variables $X, Y, E^{x}$, and $E^{y}$ span a subspace of the unconstrained phase space of the total system. The constraint $\mathcal{K}=0$, containing only these variables, can be seen as a Hamiltonian function generating a flow in this subspace along the vector field, $X \partial_{X}+Y \partial_{Y}-E^{x} \partial_{E^{x}}-$ $E^{y} \partial_{E^{y}}$. Among those functions whose Lie derivatives vanish along this vector field are $X E^{x} Y E^{y}$, which appears in the Hamiltonian constraint, and $E^{x} / E^{y}$, which is related to the "wave factor" defined in [20]. The second "no wave" constraint, $\mathcal{E}^{\prime}=0$ containing a derivative, does not have such an interpretation. 


\section{GEOMETRIC QUANTITIES}

To develop a flat-space background geometry for plane waves it is helpful to consider geometric quantities, as well as their time derivatives and interpretations, with respect to co-moving observers, i. e. we choose zero shift vectors and assume a fixed gauge of triads. This kind of evolution is generated by the Hamiltonian constraint. The time derivatives of the triad variables $\mathcal{E}, E^{x}$, and $E^{y}$ are given by Poisson brackets with $H$, in fact with the part $-\frac{1}{\kappa} H_{K}$.

Length: A local measure of length is given by

$$
\ell:=\sqrt{g_{z z}}=\sqrt{\frac{E^{x} E^{y}}{\mathcal{E}}}
$$

with the time derivative

$$
\dot{\ell}=\{\ell, H[N]\}=\frac{N}{\gamma}\left(\mathcal{A}+\eta^{\prime}\right) .
$$

A coordinate interval $\mathcal{I}$ has physical length

$$
\ell(\mathcal{I})=\int_{\mathcal{I}} \sqrt{\frac{E^{x} E^{y}}{\mathcal{E}}} \mathrm{d} z .
$$

Volume: The local measure of volume is the square root of the determinant of the spatial metric in equation (2)

$$
V=\sqrt{\mathcal{E} E^{x} E^{y}}
$$

Its time derivative is

$$
\dot{V}=\frac{N}{\gamma}\left(\frac{X E^{x}+Y E^{z}}{\mathcal{E}}+\mathcal{A}+\eta^{\prime}\right) .
$$

Cross section: The geometrical meaning of the quantity $\mathcal{E}$ is a cross section area, as $g_{x x} \cdot g_{y y}=\mathcal{E}^{2}$ is the determinant of the 2 -metric in the $(x, z)$ plane. Its time derivative is

$$
\dot{\mathcal{E}}=\frac{N}{\gamma} \frac{X E^{x}+Y E^{y}}{\ell} .
$$

The logarithmic ratio, which is also called the "wave factor" in Ref. [20],

$$
\beta=\ln \frac{E^{x}}{E^{y}}
$$

has the time derivative

$$
\dot{\beta}=\frac{N}{\gamma} \frac{Y E^{y}-X E^{x}}{\mathcal{E} \ell}
$$


Curvatures: From Ref. [8], we have the extrinsic curvature components $\left(K_{a}^{i}=e_{b}^{i} K_{a}^{b}\right)$ in terms of time derivatives

$$
\begin{aligned}
& K_{x}:=\sqrt{\left(K_{x}{ }^{1}\right)^{2}+\left(K_{x}{ }^{2}\right)^{2}}=\frac{1}{2 N} \sqrt{\frac{E^{x}}{\mathcal{E} E^{y}}} \partial_{t}\left(\mathcal{E} \frac{E^{y}}{E^{x}}\right) \\
& K_{y}:=\sqrt{\left(K_{y}{ }^{1}\right)^{2}+\left(K_{y}{ }^{2}\right)^{2}}=\frac{1}{2 N} \sqrt{\frac{E^{y}}{\mathcal{E} E^{x}}} \partial_{t}\left(\mathcal{E} \frac{E^{x}}{E^{y}}\right) \\
& K_{z} \equiv K_{z}{ }^{3}=\frac{1}{2 N} \frac{\mathcal{E}}{E^{x} E^{y}} \partial_{t}\left(\frac{E^{x} E^{y}}{\mathcal{E}}\right) .
\end{aligned}
$$

As we introduced in section II, $\gamma K_{x}=X$ and $\gamma K_{y}=Y$. Using the Hamiltonian constraint to express the time derivative we have

$$
K_{z}=\frac{1}{\gamma} \sqrt{\frac{E^{x} E^{y}}{\mathcal{E}}}\left(\mathcal{A}+\eta^{\prime}\right)=\frac{1}{\gamma} \frac{\mathcal{E}}{V}\left(\mathcal{A}+\eta^{\prime}\right)=\partial_{t} \ln \ell .
$$

The above length and time derivatives of cross section area yield a geometric interpretation of the Killing constraint $\mathcal{K}$. The expression for the Killing constraint $\mathcal{K}$

$$
\mathcal{K}=X E^{x}+Y E^{y}=\frac{\gamma}{N} \ell \dot{\mathcal{E}}
$$

has the geometrical meaning of (length) $\times($ time derivative of cross-section). We note that for a certain choice of the lapse function, namely

$$
N=\sqrt{\frac{E^{x} E^{y}}{\mathcal{E}}}=\sqrt{g_{z z}}=\ell,
$$

which is natural in the sense that it means $g_{t t}=g_{z z}, X E^{x}+Y E^{y}$ is precisely the time derivative of the cross section area. With this choice inserted into (30) we find $\mathcal{K}=\gamma \dot{\mathcal{E}}$. In $U_{ \pm}$this is set equal to $\mp \mathcal{E}^{\prime}$, which underlines the character of $U_{ \pm}$as plane wave constraints. Imposing $\mathcal{K}=0$ together with the constraint $\mathcal{E}^{\prime}=0$, meaning that the area of the cross section in the $(x, y)$ plane is constant in $z$, enforces the space-time translational invariance of the "no wave" state. This is the geometrical meaning of the Killing constraints, equations (14) and (15).

For an interpretation in terms of canonically conjugate variables it is convenient to consider the triads as configuration variables and carry out a canonical "point transformation" to the new variables $\mathcal{E}, \ell$, and $\beta$. To find new conjugate momenta, corresponding to the 
time evolution introduced above, we first express the variables $X, Y$, and $\mathcal{A}$ in terms of the time derivatives $\dot{\ell}, \dot{\mathcal{E}}$, and $\dot{\beta}$ from equations (19)), (23)), and (25)).

Using this we can construct the Lagrangian density

$$
\mathcal{L}=\dot{\mathcal{E}} \mathcal{A}+\dot{E}^{x} X+\dot{E}^{y} Y-H
$$

(Here we consider the Gauß constraint as satisfied, so that $\eta$ is irrelevant.) The kinetic part of the Lagrangian is

$$
L_{K}=\gamma \int \frac{\mathrm{d} x}{N}\left(\frac{1}{4} \frac{\ell \dot{\mathcal{E}}^{2}}{\mathcal{E}}-\frac{1}{4} \mathcal{E} \ell \dot{\beta}^{2}+\dot{\ell} \dot{\mathcal{E}}\right) .
$$

From this we can derive the conjugate momenta

$$
\begin{aligned}
& p_{\ell}=\frac{\delta L}{\delta \dot{\ell}}=\frac{\gamma}{N} \dot{\mathcal{E}}, \\
& p_{\mathcal{E}}=\frac{\delta L}{\delta \dot{\mathcal{E}}}=\frac{\gamma}{N}\left(\frac{\ell \dot{\mathcal{E}}}{2 \mathcal{E}}+\dot{\ell}\right), \\
& p_{\beta}=\frac{\delta L}{\delta \dot{\beta}}=-\frac{\gamma}{N} \frac{\mathcal{E} \ell \dot{\beta}}{2} .
\end{aligned}
$$

Using the time derivatives in terms of $X, Y$, and $\mathcal{A}$ gives the new momenta as functions of the original phase space coordinates and so completes the canonical transformation $\left(E^{x}, E^{y}, \mathcal{E} ; X, Y, \mathcal{A}\right) \leftrightarrow\left(\ell, \mathcal{E}, \beta ; p_{\ell}, p_{\mathcal{E}}, p_{\beta}\right):$

$$
\begin{aligned}
& p_{\ell}=\frac{1}{\ell}\left(X E^{x}+Y E^{y}\right), \\
& p_{\mathcal{E}}=\frac{X E^{x}+Y E^{y}}{2 \mathcal{E}}+\mathcal{A}, \\
& p_{\beta}=\frac{X E^{x}-Y E^{y}}{2} .
\end{aligned}
$$

From the first relation we see that the Killing constraint is the product of length and its conjugate momentum from equation (37),

$$
\mathcal{K}=\ell p_{\ell}
$$

which will be of some interest when we discuss fluctuations. Also note that due to the Poisson bracket relations $\{\mathcal{K}, V\}=V$ and $\{\mathcal{K}, \ell\}=\ell$ the constraint function $\mathcal{K}$ can also be interpreted as a volume and length dilatation generator. 


\section{QUANTUM STATES AND OPERATORS}

In this section we briefly present quantum states and some basic operators introduced in detail in Refs. [9, 10]. We will then apply the Killing constraints to this kinematic state space.

\section{A. Basic states}

Basic states are constructed from a one-dimensional version of spin networks, denoted as "charge-networks" in Ref. [9] with a graph $G$ comprising edges and vertices along the $z$ axis. Along an edge $e$ we define holonomies of the connection component $\mathcal{A}$,

$$
h_{e}[\mathcal{A}]=\exp \left(i \frac{k_{e}}{2} \int_{e} \mathcal{A}\right)
$$

The edge label $k_{e} \in \mathbb{Z}$ denotes a representation of $U(1)$, so the scalar density $\mathcal{A}$ appears in a natural way as a $U(1)$ connection. The connections $X$ and $Y \in \mathbb{R}$ are scalars the natural holonomies of which are point holonomies at the vertices $v$ (the location $z(v)$ of the vertex $v$ will be frequently written as $v$ )

$$
h_{v}[X]=\exp \left(i \frac{\mu_{v}}{2} X(v)\right) \quad \text { and } \quad h_{v}[Y]=\exp \left(i \frac{\nu_{v}}{2} Y(v)\right)
$$

with vertex labels $\mu_{v}$ and $\nu_{v}$ in $\mathbb{R}$. These holonomies are unitary representations of the Bohr

compactification of the reals, see Ref. [9, 19]. The angular variable $\eta \in \mathbb{R} / \mathbb{Z}$ gives rise to the point holonomies

$$
h_{v}[\eta]=\exp \left(i \lambda_{v} \eta(v)\right)
$$

in $U(1)$ with $\lambda_{v} \in \mathbb{Z}$. By application of the Gauß constraint these holonomies are expressed in terms of edge holonomies and the labels $\lambda_{v}$ can be eliminated [9]. A typical gaugeinvariant state function based on a one-dimensional graph $G$ with edges $e$ and vertices $v$ that is annihilated by the Gauß constraint is a product of the holonomies introduced above

$$
T_{G, \vec{k}, \vec{\mu}, \vec{\nu}}=\prod_{e \in G} \exp \left[i \frac{k_{e}}{2} \int_{e}\left\{\mathcal{A}(z)-\eta^{\prime}(z)\right\}\right] \prod_{v \in G} \exp \left(i \frac{\mu_{v}}{2} X\right) \exp \left(i \frac{\nu_{v}}{2} Y\right),
$$

These SNW functions, with all labels being nonzero, form an orthogonal basis of the kinematical Hilbert space. 


\section{B. Basic Operators}

The basic operators constructed from the configuration variables are holonomy operators that act on state functions.

$$
\begin{aligned}
& \hat{h}_{z}(\mathcal{I}):=\exp \left(\tau_{3} \int_{\mathcal{I}} \mathcal{A}\left(z^{\prime}\right) \mathrm{d} z^{\prime}\right)=\cos \left(\frac{1}{2} \int_{\mathcal{I}} \mathcal{A}\right)+2 \tau_{3} \sin \left(\frac{1}{2} \int_{\mathcal{I}} \mathcal{A}\right), \\
& \hat{h}_{x}(z):=\exp \left(\mu_{0} X(z) \tau_{x}(z)\right)=\cos \left(\frac{\mu_{0}}{2} X(z)\right)+2 \tau_{x}(z) \sin \left(\frac{\mu_{0}}{2} X(z)\right), \\
& \hat{h}_{y}(z):=\exp \left(\nu_{0} Y(z) \tau_{y}(z)\right)=\cos \left(\frac{\nu_{0}}{2} Y(z)\right)+2 \tau_{y}(z) \sin \left(\frac{\nu_{0}}{2} Y(z)\right) .
\end{aligned}
$$

where $\mathcal{I}$ is some interval on the $z$ axis; $\mu_{0}$ and $\nu_{0}$ are parameters that determine the representation of the holonomy to be created; the $k$-label of the edge holonomy created by $\hat{h}_{z}$ is chosen to be equal to one. The matrices $\tau_{i}=-i \sigma_{i} / 2$ are $S U(2)$ generators. For the connection $\mathcal{A}$ the generator $\tau_{3}$ is fixed, the $z$-dependent generators $\tau_{x}$ and $\tau_{y}$ are defined by

$$
\tau_{x}(z)=\cos (\eta(z)) \tau_{1}+\sin (\eta(z)) \tau_{2}, \quad \tau_{y}(z)=-\sin (\eta(z)) \tau_{1}+\cos (\eta(z)) \tau_{2} .
$$

The conjugate variables give rise to flux operators. The scalar $\mathcal{E}(z)$ at an arbitrary point $z$ acts in the following way on a state $T$

$$
\hat{\mathcal{E}}(z) T_{G, \vec{k}, \vec{\mu}, \vec{\nu}}=\frac{\gamma \ell_{\mathrm{P}}^{2}}{2} \frac{k_{+}(z)+k_{-}(z)}{2} T_{G, \vec{k}, \vec{\mu}, \vec{\nu}}
$$

where $k_{ \pm}(z)$ denotes the edge labels on the two edges meeting at $z$, if there is a vertex, or the edge label of one edge if there is no vertex. (In this case $\left(k_{+}+k_{-}\right) / 2=k(z)$.) The fundamental length scale is set by $\ell_{\mathrm{P}}^{2}=\kappa \hbar$.

The scalar densities $E^{x}, E^{y}$ have to be integrated over an interval $\mathcal{I}$ to give the operators

$$
\begin{aligned}
& \mathcal{F}_{x}(\mathcal{I})=\int_{\mathcal{I}} \hat{E}^{x} T_{G, \vec{k}, \vec{\mu}, \vec{\nu}}=\frac{\gamma \ell_{\mathrm{P}}^{2}}{2} \sum_{v \in \mathcal{I}} \mu_{v} T_{G, \vec{k}, \vec{\mu}, \vec{\nu}}, \\
& \mathcal{F}_{y}(\mathcal{I})=\int_{\mathcal{I}} \hat{E}^{y} T_{G, \vec{k}, \vec{\mu}, \vec{\nu}}=\frac{\gamma \ell_{\mathrm{P}}^{2}}{2} \sum_{v \in \mathcal{I}} \nu_{v} T_{G, \vec{k}, \vec{\mu}, \vec{\nu}} .
\end{aligned}
$$

Obviously, the flux operators are diagonal in the SNW basis.

\section{Geometric operators}

The classical geometric quantities volume and length may be quantized straightforwardly using LQG methods. 
Volume: Classically the volume of a block of space, bounded by planes of unit coordinate area in the $x$ and $y$ directions, is, from equation (2)

$$
V(\mathcal{I})=\int_{\mathcal{I}} \mathrm{d} z \sqrt{g}=\int_{\mathcal{I}} \mathrm{d} z \sqrt{\mathcal{E} E^{x} E^{y}}
$$

over an interval $\mathcal{I}$ of coordinate length $\epsilon$. All the classical triad variables are positive, $E^{x}$ and $E^{y}$ are radial variables, and so $\mathcal{E}$ must be positive as long as the sign of the spatial metric does not change. In quantum theory we allow for both signs and take the absolute values in the volume. If $\mathcal{I}$ contains one vertex, we have

$$
\begin{gathered}
V(\mathcal{I}) \approx \epsilon \sqrt{\left|\mathcal{E}(v) E^{x}(v) E^{y}(v)\right|}=\sqrt{|\mathcal{E}(v)|} \sqrt{\epsilon\left|E^{x}(v)\right|} \sqrt{\epsilon\left|E^{y}(v)\right|}= \\
\sqrt{|\mathcal{E}(v)|} \sqrt{\left|\int_{\mathcal{I}} E^{x}\right|} \sqrt{\left|\int_{\mathcal{I}} E^{y}\right|} .
\end{gathered}
$$

Inserting the corresponding flux operators and letting the resulting volume operator $\hat{V}(\mathcal{I})$ act on a vertex function of a SNW state, defined by

$$
|v\rangle:=\left|k_{ \pm}, \mu_{v}, \nu_{v}\right\rangle
$$

gives $\hat{V}(\mathcal{I})|v\rangle=V_{v}|v\rangle$ with the eigenvalue

$$
V_{v}=\frac{\gamma^{\frac{3}{2}} \ell_{\mathrm{P}}^{3}}{4} \sqrt{\left|k_{v}\right|\left|\mu_{v}\right|\left|\nu_{v}\right|}
$$

where $k_{v}:=k_{+}+k_{-}$is the sum of the labels of the two adjacent edges.

Length: Analogously we may introduce a $z$-length operator, starting from the classical length of an interval $\mathcal{I}$. Unlike the volume, this expression for length contains $\mathcal{E}$ in the denominator. As the flux operator $\hat{\mathcal{E}}$ does not have a densely defined inverse, we first replace the expression in equation (20) by applying Thiemann's identity [19] involving the Poisson bracket of quantities that have a direct operator meaning. With the holonomy (45) we find, for small intervals $\mathcal{I}$,

$$
h_{z}(\mathcal{I})\left\{h_{z}^{-1}(\mathcal{I}), V\right\} \approx-\gamma \kappa \frac{\tau_{3}}{2} \sqrt{\frac{E^{x} E^{y}}{\mathcal{E}}} .
$$

After quantization, when the Poisson bracket is replaced by $(i \hbar)^{-1}$ times the commutator of the corresponding operators, we conclude that the quantum operator of length can be written as

$$
\hat{\ell}(\mathcal{I})=\operatorname{Tr}\left[\frac{-4 i}{\gamma \ell_{\mathrm{P}}^{2}} \hat{h}_{z}(\mathcal{I})\left[\hat{h}_{z}^{-1}(\mathcal{I}), \hat{V}(\mathcal{I})\right] \tau_{3}\right]
$$


When applied to an interval $\mathcal{I}$ with one vertex $v, \hat{\ell}(\mathcal{I})$ gives the eigenvalue

$$
\ell_{v}=\frac{\sqrt{\gamma} \ell_{\mathrm{P}}}{\sqrt{2}} \sqrt{\left|\mu_{v}\right|\left|\nu_{v}\right|}\left(\sqrt{\left|k_{v}+1\right|}-\sqrt{\left|k_{v}-1\right|}\right) .
$$

When the edge labels $k_{+}$and $k_{-}$are large, $\sqrt{k_{v}+1}-\sqrt{k_{v}-1} \approx 1 / \sqrt{k_{v}}$. So when the values $\mu_{v}$ and $\nu_{v}$ at a vertex are fixed and the sum of the edge labels is sent off toward infinity the length eigenvalues can become arbitrarily small. In this limit $\ell_{v}$ becomes proportional to $V_{v} / k_{v}$. This means that the length of a $z$-interval as the thickness of a block of space in the $(x, y)$ plane is approximately the block's volume divided by the $(x, y)$-area, given by the eigenvalue of the flux operator $\hat{\mathcal{E}}$ (49]) [26].

Inverse volume: The quantization of the $V^{-1}$ operator proceeds by re-writing it in terms of the well-defined classical quantities as done in [9]

$$
V(I)^{-1}=\frac{16}{81 \kappa^{3} \gamma^{3} \mu_{0} \nu_{0}} \epsilon^{a b c} \operatorname{Tr}\left[h_{a}\left\{h_{a}^{-1}, V^{\frac{1}{3}}\right\} h_{b}\left\{h_{b}^{-1}, V^{\frac{1}{3}}\right\} h_{c}\left\{h_{c}^{-1}, V^{\frac{1}{3}}\right\}\right]
$$

So the quantum version is defined as

$$
\widehat{V^{-1}}=-\frac{16 i}{81 \hbar^{3} \kappa^{3} \gamma^{3} \mu_{0} \nu_{0}} \epsilon^{a b c} \operatorname{Tr}\left[\hat{h}_{a}\left[\hat{h}_{a}^{-1}, \hat{V}^{\frac{1}{3}}\right] \hat{h}_{b}\left[\hat{h}_{b}^{-1}, \hat{V}^{\frac{1}{3}}\right] \hat{h}_{c}\left[\hat{h}_{c}^{-1}, \hat{V}^{\frac{1}{3}}\right]\right]
$$

where $a, b$, and $c$ are summed over $x, y, z$. The action of this operator on a vertex is given in Appendix $\mathrm{A}$ with the result that

$$
\begin{array}{r}
\widehat{V^{-1}}\left|k_{ \pm}, \mu_{v}, \nu_{v}\right\rangle=V_{v}^{-1}\left|k_{ \pm}, \mu_{v}, \nu_{v}\right\rangle \text { with } \\
V_{v}^{-1}=\frac{-1}{108 \ell_{P}^{3} \gamma^{\frac{3}{2}} \mu_{0} \nu_{0}} \sqrt[3]{\left|k_{v} \mu_{v} \nu_{v}\right|}\left(\sqrt[6]{\left|\mu_{v}-\mu_{0}\right|}-\sqrt[6]{\left|\mu_{v}+\mu_{0}\right|}\right) \\
\times\left(\sqrt[6]{\left|\nu_{v}-\nu_{0}\right|}-\sqrt[6]{\left|\nu_{v}+\nu_{0}\right|}\right)\left(\sqrt[6]{\left|k_{v}-1\right|}-\sqrt[6]{\left|k_{v}+1\right|}\right) .
\end{array}
$$

Cross section: Given the simple form of the cross section operator its quantization is immediate. At a vertex

$$
\hat{\mathcal{E}}\left|k_{ \pm}, \mu_{v}, \nu_{v}\right\rangle=\left(\frac{\gamma \ell_{\mathrm{P}}^{2}}{4}\right) k_{v}\left|k_{ \pm}, \mu_{v}, \nu_{v}\right\rangle
$$

Extrinsic Curvatures and zero curvature states: Using the holonomies of equations (46,47), the quantization of the $x$ extrinsic curvature is,

$$
\widehat{K}_{x}:=\frac{2}{\mu_{0}} \operatorname{Tr}\left[\tau_{x}\left(\hat{h}_{x}(z)-\hat{h}_{x}^{-1}(z)\right)\right]
$$


The action at a single vertex is given by

$$
\widehat{K}_{x}\left|k_{ \pm}, \mu_{v}, \nu_{v}\right\rangle=-\frac{i}{\kappa \gamma^{2} \mu_{0}}\left(\left|k_{ \pm}, \mu_{v}+\mu_{0}, \nu_{v}\right\rangle-\left|k_{ \pm}, \mu_{v}-\mu_{0}, \nu_{v}\right\rangle\right)
$$

One could attempt to model flat space by requiring vertex states to satisfy

$$
\widehat{K}_{x} \sum_{\mu_{v}} a_{\mu_{v}}\left|k_{ \pm}, \mu_{v}, \nu_{v}\right\rangle=0
$$

However this requires constant coefficients, $a_{\mu_{v}+\mu_{0}}=a_{\mu_{v}-\mu_{0}}$, so such solutions are nonnormalizable and not in the kinematical Hilbert space.

The curvature operator in the $z$-direction has an ordering ambiguity between the $z$ holonomy, triad $\mathcal{E}$, and the inverse volume operator. However, there is only one Hermitian ordering of these quantities. For short intervals $\mathcal{I}$ we approximate

$$
\left(\mathcal{A}+\eta^{\prime}\right) \approx 2 \operatorname{Tr}\left[\tau_{3}\left(h_{z}(\mathcal{I})-h_{z}^{-1}(\mathcal{I})\right)\right]
$$

and use this to define the quantum operator

$$
\widehat{K}_{z}:=2 i\left(\hat{\mathcal{E}} \widehat{V^{-1}}\right)^{1 / 2} \operatorname{Tr}\left[\tau_{3}\left(h_{z}(\mathcal{I})-h_{z}^{-1}(\mathcal{I})\right)\right]\left(\hat{\mathcal{E}} \widehat{V^{-1}}\right)^{1 / 2}
$$

The operator $\hat{K}_{z}$ has the action

$$
\begin{aligned}
\widehat{K}_{z}\left|k_{ \pm}, \mu_{v}, \nu_{v},\right\rangle= & \mathcal{R}\left(\mu_{v}, \nu_{v}\right)\left|k_{v}\right|^{2 / 3}\left(\sqrt[6]{\left|k_{v}+1\right|}-\sqrt[6]{\left|k_{v}-1\right|}\right)^{1 / 2} \\
& \times\left[\left|k_{v}+1\right|^{2 / 3}\left(\sqrt[6]{\left|k_{v}+2\right|}-\sqrt[6]{\left|k_{v}\right|}\right)\left|k_{ \pm}+1, \mu_{v}, \nu_{v}\right\rangle\right. \\
& \left.-\left|k_{v}-1\right|^{2 / 3}\left(\sqrt[6]{\left|k_{v}\right|}-\sqrt[6]{\left|k_{v}-2\right|}\right)\left|k_{ \pm}-1, \mu_{v}, \nu_{v}\right\rangle\right]
\end{aligned}
$$

where

$$
\mathcal{R}(\mu, \nu):=\frac{i}{648 \mu_{0} \nu_{0}} \sqrt[3]{|\mu \nu|}\left(\sqrt[6]{\left|\mu-\mu_{0}\right|}-\sqrt[6]{\left|\mu+\mu_{0}\right|}\right)\left(\sqrt[6]{\left|\nu-\nu_{0}\right|}-\sqrt[6]{\left|\nu+\nu_{0}\right|}\right)
$$

and $k_{ \pm}+1\left(k_{ \pm}-1\right)$ mean that both $k_{+}$and $k_{-}$are raised (or lowered) by one on the intersection of the interval $\mathcal{I}$ with the two adjacent edges of the vertex $v$.

For vanishing $z$ extrinsic curvature we consider (non-degenerate) states such that, at every vertex,

$$
\widehat{K}_{z} \sum_{k_{v}} a_{k_{v}}\left|k_{v}, \mu_{v}, \nu_{v}\right\rangle=0
$$


Equation (66) allows us to establish a recursion relation between $a_{k_{v}+1}$ and $a_{k_{v}-1}$

$$
a_{k_{v}+1}=\left[\left(\frac{\left|k_{v}-1\right|}{\left|k_{v}+1\right|}\right)\left(\frac{\sqrt[6]{\left|k_{v}\right|}-\sqrt[6]{\left|k_{v}-2\right|}}{\sqrt[6]{\left|k_{v}+2\right|}-\sqrt[6]{\left|k_{v}\right|}}\right)\right]^{1 / 2} a_{k_{v}-1}
$$

This recursion relation iterates easily so that after $m$ terms

$$
a_{k_{v}+2 m+1}=\left[\left(\frac{\left|k_{v}-1\right|}{\left|k_{v}+2 m+1\right|}\right)\left(\frac{\sqrt[6]{\left|k_{v}\right|}-\sqrt[6]{\left|k_{v}-2\right|}}{\sqrt[6]{\left|k_{v}+2 m+2\right|}-\sqrt[6]{\left|k_{v}+2 m\right|}}\right)\right]^{1 / 2} a_{k_{v}-1}
$$

For large $m$ then the coefficients scale as

$$
a_{k+2 m+1} \propto m^{-\frac{1}{12}}
$$

which does not converge fast enough to ensure normalization.

We see that constraining any of the three extrinsic curvature operators to vanish yields non-nomalizable states in the kinematic Hilbert space. For this reason in the next section we will formulate flatness using the Killing constraints which (eventually) yield normalizable solutions.

\section{The Hamiltonian Constraint Operator $\hat{H}_{K}^{1}$}

The formulation of the Killing constraint requires the first part of the Hamiltonian constraint operator. Our quantization is similar to Ref. [10], which is close to the construction employed in full LQG, where the Hamiltonian constraint is regularized in form of holonomies. Following this method we arrive at a slightly different operator than in Ref. [9].

The details are given in Appendix B with the result that, on gauge invariant states, $\left|k_{ \pm}, \mu_{v}, \nu_{v}\right\rangle$

$$
\hat{H}_{K}^{1}[N]\left|k_{ \pm}, \mu_{v}, \nu_{v}\right\rangle=\frac{1}{\sqrt{2} \gamma^{2} \mu_{0} \nu_{0}} \sum_{v} N_{v} \ell_{v} \sin \left(\mu_{0} X\right) \sin \left(\nu_{0} Y\right)\left|k_{ \pm}, \mu_{v}, \nu_{v}\right\rangle .
$$

(Up to a factor 2, this is equation (55) of Ref. [9].) $N_{v}$ is the value of the lapse function at the vertex $v$. At each vertex this term alters the labels $\mu_{v}$ and $\nu_{v}$ by $\pm \mu_{0}$ and $\pm \nu_{0}$, respectively; it does not create new vertices. Whereas in full theory it appears natural that the Hamiltonian constraint changes the spin weights of edge holonomies by $\pm 1 / 2$, there is no a priori natural choice for $\mu_{0}$ and $\nu_{0}$ in the point holonomies, which are in fact artifacts of the symmetry reduction. 
We have seen that "no-curvature" constraints yield non-normalizable states. In the next sections we formulate and implement the Killing constraints. This proves to be not a simple matter of imposing the constraints, at least when we also ask that the expectation values of length and volume on an atom of geometry be finite, but requires a re-formulation of the Killing constraint.

\section{FORMULATING THE KILLING CONSTRAINT}

Like the Hamiltonian constraint, the Killing constraint $\mathcal{K}$ in the form of equation (14) contains connection variables that do not have a direct meaning as operators. A substitute is easily found in form of the Poisson bracket between the well-defined volume operator and the first part $H_{K}^{1}$ of the Hamiltonian constraint. Locally we have

$$
\mathcal{K}(z)=\frac{2}{\kappa \gamma}\left\{\frac{X E^{x} Y E^{y}}{\sqrt{\mathcal{E} E^{x} E^{y}}}(z), V(\mathcal{I})\right\}=2 \frac{\gamma}{\kappa}\left\{H_{K}^{1}(z), V(\mathcal{I})\right\} .
$$

In consequence, the first version of the Killing constraint $\hat{\mathcal{K}}$ can be defined as the corresponding commutator

$$
\hat{\mathcal{K}}:=i\left[\hat{H}_{K}^{1}, \hat{V}(\mathcal{I})\right]
$$

Note that we define the operator without the factor of 2. Since the action of the operator will vanish on states we also set $\gamma$ and the Planck length to 1 for the remainder of the paper.

The constraint turns out to act on each vertex individually as $\hat{\mathcal{K}}|v\rangle=0$. Before obtaining solutions, we note that for each vertex function $|v\rangle$ the solutions, although being normalizable in the kinematic Hilbert space, yields diverging expectation values for volume and length, as will be shown in Section VI. For this reason we explore modifications and generalizations of the above operator.

We can multiply $\mathcal{K}$ with an arbitrary positive power of the volume, supposing the volume and length contribution of any vertex are non-zero (justified later on in quantum theory). Using the same algebra as equation (73),

$$
V^{n-1}(\mathcal{I}) \mathcal{K}(z)=\frac{2}{n}\left\{H_{K}^{1}(z), V^{n}(\mathcal{I})\right\}, \quad n \geq 1,
$$

with $\mathcal{K}$ arising from $n=1$. 
A similar modification of the Killing constraint can be brought about by a modification of $H_{K}^{1}$, denoted by

$$
\left(H_{K}^{1}\right)_{p}:=-2 \operatorname{Tr}\left[\left(h_{x} h_{y} h_{x}^{-1} h_{y}^{-1}-h_{y} h_{x} h_{y}^{-1} h_{x}^{-1}\right) h_{z}\left\{h_{z}^{-1}, V^{p}(\mathcal{I})\right\}\right]
$$

which, to leading order, is

$$
p X E^{x} Y E^{y} V^{p-2}=p H_{K}^{1} V^{p-1}
$$

(The original $H_{K}^{1}$ is obtained by setting $p=1$.) The action of the corresponding operator on a vertex state is

$$
\left(\hat{H}_{K}^{1}\right)_{p}|v\rangle=\frac{p}{2 \mu_{0} \nu_{o}}\left[\left(\left|\mu_{v}\right|\left|\nu_{v}\right|\right)^{\frac{p}{2}}\left(\left|k_{v}+2\right|^{\frac{p}{2}}-\left|k_{v}-2\right|^{\frac{p}{2}}\right) \sin \left(\mu_{0} X\right) \sin \left(\nu_{0} Y\right)\right]|v\rangle .
$$

The Poisson bracket of $V^{q}$ with (77) (omitting the pre-factors) gives

$$
\left\{V^{q}, X E^{x} Y E^{y} V^{p-2}\right\}=\frac{q}{2} V^{p+q-2}\left(X E^{x}+Y E^{y}\right) .
$$

So for a given expression $V^{n} \mathcal{K}$ with $n=p+q-2$ there is a two-parameter family of

inequivalent commutators $\left[\left(\hat{H}_{K}^{1}\right)_{p}, \hat{V}^{q}\right]$ corresponding to equation (79) . They give rise to a two-parameter family of modified operators, depending on $p$ and $q$, which will be denoted by the "volume weighted" Killing constraint

$$
\hat{\mathcal{K}}_{p, q}:=i\left[\left(\hat{H}_{K}^{1}\right)_{p}, \hat{V}^{q}\right]
$$

with $\mathcal{K}_{1,1}=\mathcal{K}$. The meaning of these modifications will be clear when we construct solutions to the Killing constraint.

\section{IMPLEMENTING QUANTUM KILLING CONSTRAINTS}

The constraint $\mathcal{E}^{\prime}=0$ is easy to handle as an operator. When the scalar density $\mathcal{E}^{\prime}$, respectively the operator density $\hat{\mathcal{E}}^{\prime}$, is integrated over an interval $\mathcal{I}$, we obtain the flux operator (see equation (49))

$$
\int_{\mathcal{I}} \hat{\mathcal{E}}^{\prime} \mathrm{d} z=\hat{\mathcal{E}}_{+}-\hat{\mathcal{E}}_{-}
$$

where $\hat{\mathcal{E}}_{+}$and $\hat{\mathcal{E}}_{-}$mean the operators $\hat{\mathcal{E}}$ at the endpoints of the interval $\mathcal{I}$. Imposed as a local constraint on SNW states, the solutions are simply states with constant edge labels $k$.

The first version of the constraint $\mathcal{K}$ was represented as a Poisson bracket in equation (73). This will be studied in detail since this motivates the volume-weighted form of equation (79)). 
Integrating $\mathcal{K}$ over an interval $\mathcal{I}$ gives a well-defined operator. When $\mathcal{I}$ contains a vertex, the action of the corresponding operator is nontrivial. With the sine functions expanded, the operator has the action

$$
\begin{aligned}
& \hat{\mathcal{K}}\left|\mu_{v}, \nu_{v}\right\rangle=\frac{1}{\mu_{0} \nu_{0}} \sqrt{\left|\mu_{v}\right|\left|\nu_{v}\right||k|}(\sqrt{|k+1|}-\sqrt{|k-1|}) \times \\
& \quad\left\{\left(\sqrt{\left|\mu_{v}+2 \mu_{0}\right|\left|\nu_{v}+2 \nu_{0}\right|}-\sqrt{\left|\mu_{v}\right|\left|\nu_{v}\right|}\right)\left|\mu_{v}+2 \mu_{0}, \nu_{v}+2 \nu_{0}\right\rangle-\right. \\
& \quad\left(\sqrt{\left|\mu_{v}-2 \mu_{0}\right|\left|\nu_{v}+2 \nu_{0}\right|}-\sqrt{\left|\mu_{v}\right|\left|\nu_{v}\right|}\right)\left|\mu_{v}-2 \mu_{0}, \nu_{v}+2 \nu_{0}\right\rangle- \\
&\left(\sqrt{\left|\mu_{v}+2 \mu_{0}\right|\left|\nu_{v}-2 \nu_{0}\right|}-\sqrt{\left|\mu_{v}\right|\left|\nu_{v}\right|}\right)\left|\mu_{v}+2 \mu_{0}, \nu_{v}-2 \nu_{0}\right\rangle+ \\
&\left.\left(\sqrt{\left|\mu_{v}-2 \mu_{0}\right|\left|\nu_{v}-2 \nu_{0}\right|}-\sqrt{\left|\mu_{v}\right|\left|\nu_{v}\right|}\right)\left|\mu_{v}-2 \mu_{0}, \nu_{v}-2 \nu_{0}\right\rangle\right\} .
\end{aligned}
$$

Since the edge labels $k$ are not changed we abbreviate the labels in the remainder of this section and write simply $\left|\mu_{v}, \nu_{v}\right\rangle$. Introducing $m_{v}:=\frac{\mu_{v}}{\mu_{0}}$ and $n_{v}:=\frac{\nu_{v}}{\nu_{0}}$ we find

$$
\begin{aligned}
& \sqrt{\left|m_{v}\right|\left|n_{v}\right||k|}(\sqrt{|k+1|}-\sqrt{|k-1|}) \times \\
& \left\{\left(\sqrt{\left|m_{v}+2\right|\left|n_{v}+2\right|}-\sqrt{\left|m_{v}\right|\left|n_{v}\right|}\right)\left|\mu_{v}+2 \mu_{0}, \nu_{v}+2 \nu_{0}\right\rangle-\right. \\
& \left(\sqrt{\left|m_{v}-2\right|\left|n_{v}+2\right|}-\sqrt{\left|m_{v}\right|\left|n_{v}\right|}\right)\left|\mu_{v}-2 \mu_{0}, \nu_{v}+2 \nu_{0}\right\rangle- \\
& \left(\sqrt{\left|m_{v}+2\right|\left|n_{v}-2\right|}-\sqrt{\left|m_{v}\right|\left|n_{v}\right|}\right)\left|\mu_{v}+2 \mu_{0}, \nu_{v}-2 \nu_{0}\right\rangle+ \\
& \left.\left(\sqrt{\left|m_{v}-2\right|\left|n_{v}-2\right|}-\sqrt{\left|m_{v}\right|\left|n_{v}\right|}\right)\left|\mu_{v}-2 \mu_{0}, \nu_{v}-2 \nu_{0}\right\rangle\right\}=0 .
\end{aligned}
$$

Since this equation is identical for every vertex, we omit the index $v$, and supposing that $\mu_{v}$ and $\nu_{v}$ are integer multiples of $\mu_{0}$ and $\nu_{0}$, respectively, we write the vertex state as

$$
|v\rangle=\sum_{m, n} a_{m, n}\left|m \mu_{0}, n \nu_{0}\right\rangle
$$

with coefficients $a_{m, n}$.

At each vertex the following difference equation arises from imposing the Killing constraint

$$
\begin{aligned}
& \sqrt{|m-2||n-2|}(\sqrt{|m||n|}-\sqrt{|m-2||n-2|}) a_{m-2, n-2}- \\
& \sqrt{|m+2||n-2|}(\sqrt{|m||n|}-\sqrt{|m+2||n-2|}) a_{m+2, n-2}- \\
& \sqrt{|m-2||n+2|}(\sqrt{|m||n|}-\sqrt{|m-2||n+2|}) a_{m-2, n+2}+ \\
& \sqrt{|m+2||n+2|}(\sqrt{|m||n|}-\sqrt{|m+2||n+2|}) a_{m+2, n+2}=0
\end{aligned}
$$


This equation applies to the (rescaled) vertex labels $m$ and $n$ and connects quadruples of coefficients $a_{m, n}$. Due to the constant nature of the edge labels, $k$ we have omitted the factors depending on these labels.

Before considering solutions, we observe that due to the occurrence of absolute values in the length and volume eigenvalues there is some degeneracy: they do not depend on the signs of $\mu_{v}$ and $\nu_{v}$, and the signs of $m$ and $n$. Thus, we can characterize an "orientation" of basic states: $(+)$ if $\operatorname{sgn}\left(\mu_{v}\right)=\operatorname{sgn}\left(\nu_{v}\right)$ and $(-)$ if $\operatorname{sgn}\left(\mu_{v}\right)=-\operatorname{sgn}\left(\nu_{v}\right)$. (A similar orientation plays a role in loop quantum cosmology [18].) As we will see below, it is possible to construct states with pure $(+)$ or $(-)$ orientation. In the following we concentrate on positively oriented states. (The question of whether orientation is conserved under the evolution generated by the Hamiltonian constraint will be left to future work.)

To exhibit $a$ solution of $a_{m, n}$ to the Killing constraint we begin with inserting $m=n=0$ into equation (85), which leads to

$$
a_{-2,-2}-a_{2,-2}-a_{-2,2}+a_{2,2}=0
$$

Two of the coefficients can be chosen to be zero. We choose $a_{-2,2}=a_{2,-2}=0$ and find

$$
a_{-2,-2}=-a_{2,2}
$$

For $m=4, n=0$ we have

$$
a_{2,-2}-3 a_{6,-2}-a_{2,2}+3 a_{6,2}=0
$$

and, with the choice $a_{6,-2}=0$ we get

$$
a_{6,2}=\frac{1}{3} a_{2,2}
$$

Continuing in this way by setting $a_{m,-2}=0$ for $m>0$, we obtain the nonzero coefficients

$$
a_{4 r+2,2}=\frac{1}{2 r+1} a_{2,2}
$$

for $r \geq 0$.

Setting also $a_{m, 2}=0$ for $m<0$ and $a_{2, n}=0$ for $n<0$, we obtain a solution with nonzero coefficients confined to the first and third quadrants in the $(m, n)$ plane. This solution has the symmetry properties $a_{-m,-n}=-a_{m, n}$ and $a_{m, n}=a_{n, m}$. It has pure $(+)$ orientation and avoids zero volume or zero length states with $m=0$ or $n=0$. It may be characterized by 
one fundamental initial value $a_{2,2}$. Under the assumption of integer $m$ and $n$ and positive orientation there are eight linearly independent solutions with fundamental initial values $a_{1,1}, a_{1,2}, a_{1,3}, a_{2,1}, a_{3,1}, a_{2,2}, a_{2,3}$, and $a_{3,2}$.

If we admit non-integer values of $m$ and $n$, we can construct analogous oriented solutions with fundamental initial value $a_{m, n}$ with $0<m<2$ and $0<n<2$. Non-integer values of $m$ and $n$ mean that the vertex labels $\mu_{v}$ and $\nu_{v}$ are not integer multiples of the labels $\mu_{0}$ and $\nu_{0}$ in the Hamiltonian constraint. Supposing they are integer multiples renders the volume and length spectra discrete, such an assumption has the advantage to reflect the discreteness of full LQG in our 1+1 dimensional model.

Numerical calculations of this solution indicate that they are normalizable in the kinematical Hilbert space, but the volume and length expectation values (at each vertex),

$$
\left\langle V_{v}\right\rangle \propto \sum_{m, n}\left|a_{m, n}\right|^{2} \sqrt{|k||m||n|}
$$

and

$$
\left\langle\ell_{v}\right\rangle \propto \sum_{m, n}\left|a_{m, n}\right|^{2} \sqrt{|m||n|}(\sqrt{|k+1|}-\sqrt{|k-1|})
$$

(see equations (55) and (58)), diverge.

To investigate the asymptotic behavior of the solutions for large $m$ and $n$, we approximate the coefficients by a continuous function $a(m, n)$ and the difference equation (85) by a differential equation

$$
2 m \partial_{m} a+2 n \partial_{n} a+3 a=0
$$

A simple solution is

$$
a(m, n)=m^{\alpha} n^{\beta} \quad \text { where } \quad \alpha+\beta=-\frac{3}{2} .
$$

However, it is not hard to show that ensuring finite expectation value of length or volume,

$$
\sum_{m, n} \sqrt{m n}|a(m, n)|^{2}<\infty
$$

requires that $\alpha+\beta<-\frac{3}{2}$. Thus finite expectation values are not possible with the first version of the Killing constraint.

The divergence of the volume and length expectation values in a state on which the Killing constraints are exactly satisfied, indicates that such conditions are too strong for a physical quantum state. Already on the kinematical level we see that we cannot solve 
the Killing constraint equation $\hat{\mathcal{K}}|v\rangle=0$ for a kinematical vertex state $|v\rangle$ with reasonable physical properties. This is why we explore modifications.

Recall from equation (40) that $\mathcal{K}$ can be represented as the product of two canonically conjugate variables. In one-dimensional quantum mechanics wave functions on the interval $(0, \infty)$ that are annihilated by $\hat{q} \hat{p}, \hat{p} \hat{q}$ or the anticommutator $[\hat{q}, \hat{p}]_{+}$are non-normalizable, so the problems with solutions of $\hat{\mathcal{K}}|v\rangle=0$ may not come as a big surprise.

As discussed in the last section, it is possible to weaken the Killing constraint equation in order to find physically acceptable solutions. The volume-weighted Killing constraint operator $\hat{\mathcal{K}}_{p q}$ of equation (80) leads to difference equations of the form

$$
\begin{aligned}
& (|m-2||n-2|)^{\frac{p}{2}}\left((|m||n|)^{\frac{q}{2}}-(|m-2||n-2|)^{\frac{q}{2}}\right) a_{m-2, n-2}- \\
& (|m+2||n-2|)^{\frac{p}{2}}\left((|m||n|)^{\frac{q}{2}}-(|m+2||n-2|)^{\frac{q}{2}}\right) a_{m+2, n-2}- \\
& (|m-2||n+2|)^{\frac{p}{2}}\left((|m||n|)^{\frac{q}{2}}-(|m-2||n+2|)^{\frac{q}{2}}\right) a_{m-2, n+2}+ \\
& (|m+2||n+2|)^{\frac{p}{2}}\left((|m||n|)^{\frac{q}{2}}-(|m+2||n+2|)^{\frac{q}{2}}\right) a_{m+2, n+2}=0
\end{aligned}
$$

the solutions of which fall off more rapidly with growing $m$ and $n$, when $p$ and $q$ are large enough.

We consider states created as solutions of the generalized difference equation (95), first with $p+q=2$, so that all versions of the constraint operators correspond to the same classical expression $\mathcal{K}$ of equation (73) (and the right-hand side of equation (79) without the factor of volume). In the next step this condition is relaxed and cases with $p+q>2$ are considered. Concretely, we estimate three quantities: The average value

$$
\langle W\rangle=\left\langle\sqrt{\left|\frac{\mu_{v}}{\mu_{0}}\right| \cdot\left|\frac{\nu_{v}}{\nu_{0}}\right|}\right\rangle=\langle\sqrt{|m||n|}\rangle,
$$

which, for a fixed value of the edge parameter $k$, is proportional to both the length and the volume expectation values, the uncertainty

$$
\Delta W=\left(\left\langle W^{2}\right\rangle-\langle W\rangle^{2}\right)^{\frac{1}{2}}
$$

and the departure of a considered state function from being annihilated by $\hat{\mathcal{K}}$. Since the Killing constraint operator $\hat{\mathcal{K}}$ is not Hermitian we calculate, from the original Killing constraint, the moment

$$
\| \hat{\mathcal{K}}|v\rangle \|:=\left\langle v\left|\hat{\mathcal{K}}^{\dagger} \hat{\mathcal{K}}\right| v\right\rangle^{\frac{1}{2}}
$$


instead of $\left\langle\hat{\mathcal{K}}^{2}\right\rangle^{\frac{1}{2}}$, which contains positive and negative contributions.

We numerically calculated the quantities by inserting the coefficients into an $(m, n)$ diagram and forming sums over diagonals with slope -1 in the first quadrant, that is, e.g. $a_{2,2}$, $a_{2,4}+a_{4,2}, a_{2,6}+a_{4,4}+a_{6,2}$, and so on. The contributions of each diagonal can be fairly well fitted by a decreasing power function, and after summing up 10-15 of them, the remainders were estimated by integrals over the extrapolated power functions. The contributions of these integrals to the estimates of the infinite sums is of the order of a few percent.

For the original constraint $\mathcal{K}$ with $p=q=1$ the expectation value $\langle W\rangle$ diverges. Further, in all cases $p+q=2$ the uncertainties of length and volume diverge, and for $p>q$ they diverge more rapidly. For instance, with $p=3 / 2, q=1 / 2$ we have

$$
\langle W\rangle=5.41, \quad \Delta W=\infty, \quad \| \hat{\mathcal{K}}|v\rangle \|=1.05 .
$$

In fact as the parameters depart further from the "natural" values of $p=q=1$ we obtain decreasing expectation values, but $\Delta W$ always diverges. This need not necessarily disqualify a state as a physical state, but in a realistic quantum model for flat space such states can have only a tiny or zero probability measure. For $p<q$ the divergence of $\langle W\rangle$ becomes worse than for $p=q=1$.

For $p+q \geq 2$ some results are summarized in Tables I-III. From the Table I we see that with growing $p+q$ the average value $\langle W\rangle$ goes quickly to 2 , which means that $|2,2\rangle$ becomes dominant. Table II confirms this tendency. The limiting state for $p+q$ growing to infinity is the basis state $|2,2\rangle$, i. e. all coefficients vanish except $a_{2,2}$, which is equal to one. In this state $\| \hat{\mathcal{K}}|2,2\rangle||=4 \sqrt{3} \approx 6.92$, which is an upper bound on Table III and so the maximal departure of the considered states from $\hat{\mathcal{K}}|v\rangle=0$.

\begin{tabular}{c||c|c|c|c}
$q \backslash p$ & 1 & 2 & 3 & 4 \\
\hline \hline 1 & $\infty$ & 2.25 & 2.052 & 2.014 \\
\hline 2 & 2.55 & 2.065 & 2.015 & 2.0045 \\
\hline 3 & 2.13 & 2.02 & 2.005 & 2.0015 \\
\hline 4 & 2.04 & 2.007 & 2.002 & 2.0005
\end{tabular}

TABLE I: The expectation value $\langle W\rangle$ as a function of the parameters $q$ and $p$. The expectation value peaks on the $|2,2\rangle$ state as the parameters increase. 


\begin{tabular}{c||c|c|c|c}
$q^{\backslash p}$ & 1 & 2 & 3 & 4 \\
\hline \hline 1 & $\infty$ & 0.82 & 0.112 & 0.108 \\
\hline 2 & $\infty$ & 0.236 & 0.087 & 0.065 \\
\hline 3 & 0.75 & 0.103 & 0.052 & 0.038 \\
\hline 4 & 0.33 & 0.060 & 0.029 & 0.022
\end{tabular}

\begin{tabular}{c||c|c|c|c}
$q^{\backslash p}$ & 1 & 2 & 3 & 4 \\
\hline \hline 1 & 0 & 3.51 & 5.078 & 5.88 \\
\hline 2 & 2.86 & 4.87 & 5.82 & 6.31 \\
\hline 3 & 4.51 & 5.70 & 6.27 & 6.57 \\
\hline 4 & 5.47 & 6.19 & 6.54 & 6.72
\end{tabular}

TABLE II: The uncertainty $\Delta W$ as a function of the parameters $q$ and $p$. The uncertainty decreases rapidly with increasing parameters.

TABLE III: The moment $\| \hat{\mathcal{K}}|v\rangle \|$ defined in equation (98), as a function of the parameters $q$ and $p$. It is finite and bounded by $\| \hat{\mathcal{K}}|2,2\rangle \|=4 \sqrt{3} \approx 6.92$.

The volume-weighted Killing constraint does a better job of approximating the flat space limit of the plane gravitational space-time in that it has finite geometric expectation values and decreasing uncertainties (with $q$ and $p$ ). However, the non-vanishing moment $\| \hat{\mathcal{K}}|v\rangle \|$ and the non-hermiticity of the original Killing constraint leads us to consider a different formulation of the Killing constraint, which is described in the next section. In this case we can solve the constraint and perform a similar analysis of the uncertainties.

\section{A HERMITIAN KILLING CONSTRAINT OPERATOR}

As an alternative to the $\hat{\mathcal{K}}_{p, q}$ operator in the two foregoing sections a quite simple, Hermitian Killing operator can be constructed at least in the weak field limit. The construction arises from an approximation of $X$ and $Y$, which is valid classically for small $X$ and $Y$, i. e. for a weak gravitational field, and is an alternative to the Thiemann trick. We may approximate

$$
X(z) \approx \frac{2}{\mu_{0}} \operatorname{Tr}\left[\tau_{x} h_{x}^{-1}(z)\right] \approx \frac{2}{\mu_{0}} \operatorname{Tr}\left[\tau_{x}+\frac{1}{4} \mu_{0} X(z)\right],
$$

and analogously $Y$. For edge holonomies such an approximation becomes exact in the continuous limit, when edges grow arbitrarily short. For point holonomies, which are an artifact of the homogeneity in the $x$ and $y$ directions, we must assume $X \ll \frac{4}{\mu_{0}}$ in order to 
replace $X$ by the right-hand side of equation (100).

\section{A. Modified constraint operator and equation}

With the aid of equation (100), the modified Killing constraint

$$
\mathcal{K}_{q}:=V^{q} \mathcal{K} V^{q}
$$

can be arranged in the following symmetric operator form

$$
\hat{\mathcal{K}}_{q}[\mathcal{I}]=2 \int_{\mathcal{I}} \mathrm{d} z \hat{V}^{q}\left(\sqrt{\hat{E}^{x}} \frac{\operatorname{Tr}\left[\tau_{x} \hat{h}_{x}^{-1}[\mathcal{I}]\right]}{\mu_{0}} \sqrt{\hat{E}^{x}}+\sqrt{\hat{E}^{y}} \frac{\operatorname{Tr}\left[\tau_{y} \hat{h}_{y}^{-1}[\mathcal{I}]\right]}{\nu_{0}} \sqrt{\hat{E}^{y}}\right) \hat{V}^{q} .
$$

As before, we seek a superposition of vertex states such that

$$
\hat{\mathcal{K}}_{q} \sum_{\mu, \nu} a_{\mu, \nu}|k, \mu, \nu\rangle=0
$$

where the label $v$ of $\mu$ and $\nu$ has been suppressed. The analysis is similar to Section VI and the details are presented in Appendix C. It is also shown there that, like the previous formulation of the Killing constraint, this operator must be volume-weighted in order to ensure finite expectation values of length and volume. In Appendix $\mathrm{C}$ it is also shown that

$\mu$ and $\nu$ in (103) must not be even integer multiples of $\mu_{0}$ and $\nu_{0}$. For odd integer multiples the resulting normalized states are

$$
|q\rangle=\frac{2^{q}}{2^{q+1}-1} \frac{1}{\zeta(q+1)} \sum_{m, n=-\infty}^{\infty} \frac{1}{|(2 m+1)(2 n+1)|^{\frac{q+1}{2}}}|2 m+1,2 n+1\rangle,
$$

in the notation

$$
|2 m+1,2 n+1\rangle:=\left|k,(2 m+1) \mu_{0},(2 n+1) \nu_{0}\right\rangle,
$$

and where $\zeta(q)$ is the Riemann zeta function. These states have finite expectation values of length and volume only when $q>1$.

In the limit of large $q$ the state $|q\rangle$ reduces to a superposition of four states with the same length and volume expectation values,

$$
\lim _{q \rightarrow \infty}|q\rangle \longrightarrow \frac{1}{2}(|1,1\rangle+|1,-1\rangle+|-1,1\rangle+|-1,-1\rangle)
$$

and the uncertainties for length and volume go to zero, as we can see from

$$
\Delta W=2 \frac{\left[\left(2^{q}-1\right)^{2}\left(2^{q+1}-1\right)^{2} \zeta(q)^{2} \zeta(q+1)^{2}-\left(2^{q+\frac{1}{2}}-1\right)^{4} \zeta\left(q+\frac{1}{2}\right)^{4}\right]^{\frac{1}{2}}}{\left(2^{q+1}-1\right)^{2} \zeta(q+1)^{2}} .
$$




\section{B. Uncertainty in the Killing Constraint}

As in the previous section we consider the departure of our solution states from being annihilated by the original Killing constraint. The constraint $\hat{\mathcal{K}}_{q}$ is now Hermitian so it

makes sense to calculate the usual uncertainty $\left(\left\langle\hat{\mathcal{K}}^{2}\right\rangle-\langle\hat{\mathcal{K}}\rangle^{2}\right)^{\frac{1}{2}}$, where $\langle\hat{\mathcal{K}}\rangle$ is again equal to zero. In the following we consider a slight generalization, namely the uncertainty

$$
\Delta \mathcal{K}_{p}=\left\langle q\left|\hat{\mathcal{K}}_{p}^{2}\right| q\right\rangle^{\frac{1}{2}}
$$

where the parameter of the constraint (101) was denoted by $p$ and may be different from the state label $q$. The expression of the constraint is the same, we have only weighted the state and the uncertainty operator differently. This will allow us to perform three very similar calculations at once.

The operator $\hat{\mathcal{K}}_{p}$ acts on a vertex state $|k, \mu, \nu\rangle$ by replacing it by four states in the neighborhood, namely $\left|k, \mu \pm \mu_{0}, \nu \pm \nu_{0}\right\rangle$. By acting with $\hat{\mathcal{K}}_{p}$ on $|q\rangle$ in the notation of (105) we obtain from $|2 m+1,2 n+1\rangle$ and its neighboring states $|2(m \pm 1)+1,2(n \pm 1)+1\rangle$ contributions to the states

$$
|2 m, 2 n+1\rangle, \quad|2 m+2,2 n+1\rangle, \quad|2 m+1,2 n\rangle, \quad|2 m+1,2 n+2\rangle .
$$

Let's consider $|2 m, 2 n+1\rangle$ in detail. From the action of $\hat{\mathcal{K}}_{p}$ on $|2 m+1,2 n+1\rangle$ we find

$$
i\left(\frac{1}{4^{2 p}} 2^{\frac{p+1}{2}}|k|^{p}\left(\mu_{0} \nu_{0}\right)^{p}\right)|2 n+1|^{p}(|m||2 m+1|)^{\frac{p+1}{2}}|2 m, 2 n+1\rangle,
$$

and from the action on $|2 m-1,2 n+1\rangle$ we find

$$
-i\left(\frac{1}{4^{2 p}} 2^{\frac{p+1}{2}}|k|^{p}\left(\mu_{0} \nu_{0}\right)^{p}\right)|2 n+1|^{p}(|m||2 m-1|)^{\frac{p+1}{2}}|2 m, 2 n+1\rangle .
$$

The prefactor in parenthesis, which is the same in all cases, can be omitted because we are primarily interested in numerical comparisons for different parameters $q$ and $p$. This means that we are effectively studying the single vertex behavior of the states. Up to this prefactor, $\hat{\mathcal{K}}_{p}$ generates the state

$$
i|2 n+1|^{p} \beta_{2 n+1}|m|^{\frac{p+1}{2}}\left(|2 m+1|^{\frac{p+1}{2}} \alpha_{2 m+1}-|2 m-1|^{\frac{p+1}{2}} \alpha_{2 m-1}\right)|2 m, 2 n+1\rangle .
$$

where coefficients $\alpha$ and $\beta$ are solutions to the separated difference equations ([C7) and (C8). After insertion of these coefficients this becomes

$$
i|2 n+1|^{p-\frac{q+1}{2}}|m|^{\frac{p+1}{2}}\left(|2 m+1|^{\frac{p-q}{2}}-|2 m-1|^{\frac{p-q}{2}}\right)|2 m, 2 n+1\rangle .
$$


The other three states created from $|2 m+1,2 n+1\rangle$ by $\hat{\mathcal{K}}_{p}$ are

$$
\begin{aligned}
& i|2 n+1|^{p-\frac{q+1}{2}}|m+1|^{\frac{p+1}{2}}\left(|2 m+3|^{\frac{p-q}{2}}-|2 m+1|^{\frac{p-q}{2}}\right)|2 m+2,2 n+1\rangle, \\
& i|2 m+1|^{p-\frac{q+1}{2}}|n|^{\frac{p+1}{2}}\left(|2 n+1|^{\frac{p-q}{2}}-|2 n-1|^{\frac{p-q}{2}}\right)|2 m+1,2 n\rangle, \\
& i|2 m+1|^{p-\frac{q+1}{2}}|n+1|^{\frac{p+1}{2}}\left(|2 n+3|^{\frac{p-q}{2}}-|2 n+1|^{\frac{p-q}{2}}\right)|2 m+2,2 n+2\rangle .
\end{aligned}
$$

A second application of $\hat{\mathcal{K}}_{p}$ on $|q\rangle$ shifts the contributions back to the "old" places at odd multiples of $\mu_{0}$ and $\nu_{0}$ and to "new" places with both multiples of $\mu_{0}$ and $\nu_{0}$ being even, but only the former ones contribute to $\left\langle q\left|\hat{\mathcal{K}}_{p}\right| q\right\rangle$. In particular, the action of $\hat{\mathcal{K}}_{p}$ on the four states (110,111]112]113) contribute to the state $|2 m+1,2 n+1\rangle$, and multiplying this contribution by $a_{2 m+1,2 n+1}$ we get (up to an overall factor) the matrix element

$$
\begin{aligned}
\langle 2 m+ & \left.1,2 n+1\left|\hat{\mathcal{K}}_{p}^{2}\right| 2 m+1,2 n+1\right\rangle \propto \\
& |2 n+1|^{2 p-q-1}|2 m+1|^{\frac{p-q}{2}}\left[|m|^{p+1}\left(|2 m+1|^{\frac{p-q}{2}}-|2 m-1|^{\frac{p-q}{2}}\right)\right. \\
& \left.-|m+1|^{p+1}\left(|2 m+3|^{\frac{p-q}{2}}-|2 m+1|^{\frac{p-q}{2}}\right)\right] \\
& +|2 m+1|^{2 p-q-1}|2 n+1|^{\frac{p-q}{2}}\left[|n|^{p+1}\left(|2 n+1|^{\frac{p-q}{2}}-|2 n-1|^{\frac{p-q}{2}}\right)\right. \\
& \left.-|n+1|^{p+1}\left(|2 n+3|^{\frac{p-q}{2}}-|2 n+1|^{\frac{p-q}{2}}\right)\right] .
\end{aligned}
$$

In the following, the values $p=0$ and $p=-1 / 2$ will be of interest. The first value corresponds to the original, un-weighted Killing constraint $\mathcal{K}$ while the second value gives an expression that contains a factor of the momentum $\hat{p}_{\ell}$ at a single vertex [27]. Although the inverse length in the second one cannot be directly formulated as a densely defined operator, the above formula makes sense also for $p=-1 / 2$. This means that formula (114) can be extrapolated for $p=-1 / 2$ for states of the form $|q\rangle$ with $q>1$.

For $p=0$ and $q=2$ we have matrix elements

$$
\begin{aligned}
\langle 2 m+ & \left.1,2 n+1\left|\hat{\mathcal{K}}^{2}\right| 2 m+1,2 n+1\right\rangle \propto \\
& |2 n+1|^{-3}|2 m+1|^{-1}\left[|m|\left(|2 m+1|^{-1}-|2 m-1|^{-1}\right)\right. \\
& \left.-|m+1|\left(|2 m+3|^{-1}-|2 m+1|^{-1}\right)\right] \\
& +|2 m+1|^{-3}|2 n+1|^{-1}\left[|n|\left(|2 n+1|^{-1}-|2 n-1|^{-1}\right)\right. \\
& \left.-|n+1|\left(|2 n+3|^{-1}-|2 n+1|^{-1}\right)\right],
\end{aligned}
$$


their sum is numerically approximately equal to 1.052 ; for $q=4$ we find 1.610 . In the limit $q \rightarrow \infty$ only $m=n=0$ contributes and

$$
\left\langle q\left|\hat{\mathcal{K}}^{2}\right| q\right\rangle \rightarrow 4\left\langle 1,1\left|\hat{\mathcal{K}}^{2}\right| 1,1\right\rangle
$$

the limiting value $\Delta \mathcal{K}_{\infty}^{2}$ of $\left\langle 1,1\left|\hat{\mathcal{K}}^{2}\right| 1,1\right\rangle$ for $q \rightarrow \infty$ (without prefactors) is 2 . In the considered cases $q=2$ and $q=4, \Delta \mathcal{K}=0.72 \Delta \mathcal{K}_{\infty}$ and $0.90 \Delta \mathcal{K}_{\infty}$, respectively.

For $p=-\frac{1}{2}$ - recall that $\mathcal{K}_{-\frac{1}{2}}$ is proportional to $p_{\ell}$, see equation $\left.(31)\right)$ - and $q=2$ the matrix elements are

$$
\begin{aligned}
&\left\langle 2 m+1,2 n+1\left|\hat{\mathcal{K}}_{-\frac{1}{2}}^{2}\right| 2 m+1,2 n+1\right\rangle \propto \\
&|2 n+1|^{-4}|2 m+1|^{-\frac{5}{4}}\left[|m|^{\frac{1}{2}}\left(|2 m+1|^{-\frac{5}{4}}-|2 m-1|^{-\frac{5}{4}}\right)\right. \\
&\left.-|m+1|^{\frac{1}{2}}\left(|2 m+3|^{-\frac{5}{4}}-|2 m+1|^{-\frac{5}{4}}\right)\right] \\
&+|2 m+1|^{-4}|2 n+1|^{-\frac{5}{4}}\left[|n|\left(|2 n+1|^{-\frac{5}{4}}-|2 n-1|^{-\frac{5}{4}}\right)\right. \\
&\left.-|n+1|^{\frac{1}{2}}\left(|2 n+3|^{-\frac{5}{4}}-|2 n+1|^{-\frac{5}{4}}\right)\right],
\end{aligned}
$$

the sum of which is approximately 1.184, whereas for $q=4$ we have 1.689. Here the limit $q \rightarrow \infty$ is the same as for $p=0$, namely 2. As $p_{\ell}$ is proportional to $\mathcal{K}_{\frac{1}{2}}$, for $q=2$ and $q=4$ we find the ratios $\Delta p_{\ell}=0.77 \Delta p_{\ell, \infty}$ and $\Delta p_{\ell}=0.92 \Delta p_{\ell, \infty}$, respectively. Surprisingly at first sight, in the limit of vanishing length uncertainty, the uncertainty of the conjugate momentum of length in the form $\Delta \mathcal{K}_{-\frac{1}{2}}$ goes to a finite limit, apparently violating the naive uncertainty relation, $\Delta \ell \Delta p_{\ell} \geq \hbar / 2$ since $\Delta \ell \Delta p_{\ell} \rightarrow 0$ for large $q$. This apparent violation may be explained by the reformulation of the classically canonically conjugate quantities in the quantum theory.

\section{DISCUSSION}

The results of the present article are "flat" solutions to the Killing constraints, which provide a kinematic model of Minkowski space in this (1+1)-midisuperspace, and fluctuations of geometric quantities in these states. We find that solutions to the Killing constraint in its apparently most natural form are physically unacceptable - the expectation values of length and volume at every atom of geometry diverge. This suggests that the constraint, which assures flatness of space by the absence of gravitational waves, is too strong, and that 
quantum theory cannot satisfy the Killing constraints to the same extent as classical theory: There must be fluctuations and the Killing constraints can be valid only in some weaker form.

In Section $\nabla$ we formulate the first version of the Killing constraint in a straight-forward way, similar to using Thiemann's trick in the Hamiltonian constraint operator. Motivated by the resulting divergent expectation values of volume and length we weaken the quantum constraint with a factor of the volume. Additional tempering is achieved by introducing the modified operator $\left(\hat{H}_{K}^{1}\right)_{p}$ of equation (78) into the construction of the constraint operator defined in equation (80). In this way we obtain a two-parameter family $\hat{\mathcal{K}}_{p, q}$ of volumeweighted constraints and can construct a two-parameter family of corresponding solutions that are numerically estimated and discussed in Section VI.

We find that two cases of weighting with volume are distinguished. When $p+q=2$, the classical constraint functions are equivalent to the original form of $\mathcal{K}$. Nevertheless, the quantum operators $\hat{\mathcal{K}}_{p, q}$ act in different ways on SNW states. When $p>q$, volume and length expectation values are finite and the norm of $\hat{\mathcal{K}}|v\rangle$ is relatively small, but the uncertainties in length and volume are divergent (i.e. $\Delta W$ diverges). For this reason solutions of these modified constraints are not sufficient to model quantum flat space.

With divergent uncertainties of length and volume on a single atom of geometry we generalize to the second case with volume weighting such that $p+q>2$, a true weakening of the constraint already at the classical level. For a growing sum $p+q$ the volume and length uncertainties quickly decrease but $\Delta \mathcal{K}$ grows. Notably, as $\Delta W$ becomes smaller and smaller $\Delta \mathcal{K}$ does not grow to infinity, as one might expect from an uncertainty argument, but approaches a finite limit, namely the value for the single fundamental SNW state on which the solution is based $(|2,2\rangle$ in our explicit example in Section VI). The quantity $\Delta \mathcal{K}$ remains finite in the limit $p+q \rightarrow \infty$. The width of this residual spread of the constraint leads us to investigate another form of the Killing constraint.

This second regularization, discussed in Section VII, is motivated by the observation that, unlike the standard constraints of canonical general relativity, the Killing constraints are not symmetry generators of the whole theory, but physical conditions that pick out certain states from a larger set of states. With the physical interpretation of the classical Killing constraint $\mathcal{K}$ as the rate of change $\dot{\mathcal{E}}$ of cross section areas in the homogenous $(x, y)$ plane times the length of an atom of geometry, it makes sense to look for a Hermitian version of $\hat{\mathcal{K}}$. This 
is done in the second version that is valid when $X \ll \frac{4}{\mu_{0}}$. There is a one-parameter family of operators $\mathcal{K}_{q}$ which have essentially unique solutions (as long as $\mu_{v}$ and $\nu_{v}$ are integer multiples of $\mu_{0}$ and $\nu_{0}$ ). These solutions are derived in Appendix Cand displayed in equation (104).

In spite of the different regularizations, the two versions of the Killing constraint operator require tempering by volume and lead to qualitatively similar solutions and fluctuations, although the volume-weighted Hermitian version does not have divergent uncertainties in geometric quantities. Instead, length and volume on a single atom of geometry have finite expectation values for all values of the parameter $q>1$. The uncertainties in geometric quantities decrease with increasing $q$. The Hermitian Killing constraint has smaller spread in the uncertainty of the constraint $\Delta \mathcal{K}$ leading to qualitative improvement. Both solutions of the volume-weighted Killing constraints have vanishing expectation value for the extrinsic curvatures given in Section IVC. The existence of the analytic solutions to the Hermitian Killing constraint is the most important difference between the two versions.

We can interpret these results as follows: Metric variables like length and volume are constructed from triad variables alone, whereas in the present approach flatness is formulated in terms of Killing vectors that contain connection variables. As one can expect in quantum theory, when the constraint on one variable is relaxed, so that its uncertainty becomes larger, the uncertainty of another variable, which is conjugate or at least related to conjugate variables, becomes smaller. However, the solutions to the Hermitian Killing constraint in the limit of vanishing length (and volume) uncertainties have finite uncertainty in the length momentum $p_{\ell}$. The reason is that we reformulated the classical quantities $\ell$ and $p_{\ell}$ on their way from classical expressions to well-defined operators. In equation (57) $\ell$ is defined in terms of holonomy and volume operators, whereas $\hat{p}_{\ell}$ is defined with the aid of the commutator $\left[\hat{\mathcal{E}}, \hat{H}_{K}\right]$. In this way the commutator algebra of quantum gravity operators on some configuration spaces is not always isomorphic to the Poisson bracket algebra of the corresponding classical quantities and quantum uncertainty relations can deviate from a priori expectations. One could speculate that Planck scale modifications of quantum uncertainties, discussed in the literature as "Generalized Uncertainty Principle" (see, e.g. [21 23]), might have roots in canonical quantum gravity.

Additionally, the null Killing constraints are restrictions on time evolution so "flatness" has a space-time character. Hence, in carrying over perfect flatness in this sense from classical 
theory to quantum theory by setting the action of the unmodified Killing constraints on states to zero makes the uncertainty (and even expectation values) of triad variables at every atom of geometry infinite.

There remains much work to do to address our goal of ascertaining the effects of underlying fundamental geometric discreteness of LQG on the propagation of gravitational waves. Most immediately we need to find the physical states - states that satisfy the Hamiltonian constraint - of Minkowski space and and of the uni-directional wave space-times, and to show that the constraint algebra contains no anomalies. Work on this is underway.

Acknowledgements Work of JA was supported, in part, by the Gorin Foundation Fund of Hamilton College. We thank the referees for helpful suggestions.

\section{Appendix A: Inverse volume quantization action}

We evaluate the action of the $\widehat{V^{-1}}$ operator on our local eigenstate $|v\rangle$. Observe that, from equation (45) and the analogous identities for the $x$ and $y$ holonomies,

$$
\hat{h}_{a}^{ \pm 1}=\left(\frac{1}{2} \mp i \tau_{a}\right) \hat{e}_{a}^{+}+\left(\frac{1}{2} \pm i \tau_{a}\right) \hat{e}_{a}^{-},
$$

where $e_{a}^{ \pm}$are operators with the following actions on our eigenstates

$$
\hat{e}_{z}^{ \pm}|k, \mu, \nu\rangle=|k \pm 1, \mu, \nu\rangle
$$

and

$$
\hat{e}_{x}^{ \pm}|k, \mu, \nu\rangle=\left|k, \mu \pm \mu_{o}, \nu\right\rangle
$$

similarly for $\hat{e}_{y}^{ \pm}$. The following equalities hold

$$
\left(\frac{1}{2} \pm i \tau_{a}\right)^{2}=\left(\frac{1}{2} \pm i \tau_{a}\right)
$$

and

$$
\left(\frac{1}{2} \pm i \tau_{a}\right)\left(\frac{1}{2} \mp i \tau_{a}\right)=0
$$

Also,

$$
\hat{V}^{\frac{1}{3}}\left|k_{ \pm}, \mu_{v}, \nu_{v}\right\rangle=\frac{\left(\gamma \ell_{P}^{2}\right)^{\frac{1}{2}}}{4^{\frac{1}{3}}} \sqrt[6]{\left|\mu_{v} \nu_{v} k_{v}\right|}\left|k_{ \pm}, \mu_{v}, \nu_{v}\right\rangle
$$


For simplicity we set $\mu_{0}=1=\nu_{0}$ in the following. Therefore, the action of the factors are

$$
\begin{aligned}
\hat{h}_{a}\left[\hat{h}_{a}^{-1}, \hat{V}^{\frac{1}{3}}\right]|\bar{a}\rangle=\hat{h}_{a}\left[\left(\frac{1}{2}+i \tau_{a}\right)\left(\hat{e}_{a}^{+} \hat{V}^{\frac{1}{3}}-\hat{V}^{\frac{1}{3}} \hat{e}_{a}^{+}\right)+\left(\frac{1}{2}-i \tau_{a}\right)\left(\hat{e}_{a}^{-} \hat{V}^{\frac{1}{3}}-\hat{V}^{\frac{1}{3}} \hat{e}_{a}^{-}\right)\right]|\bar{a}\rangle \\
=\hat{h}_{a} \frac{\left(\gamma \ell_{P}^{2}\right)^{\frac{1}{2}}}{4^{\frac{1}{3}}} \sqrt[6]{|b c|}\left[\left(\frac{1}{2}+i \tau_{a}\right)(\sqrt[6]{|a|}-\sqrt[6]{|a+1|})|\bar{a}+1\rangle\right. \\
\left.\quad+\left(\frac{1}{2}-i \tau_{a}\right)(\sqrt[6]{|a|}-\sqrt[6]{|a-1|})|\bar{a}-1\rangle\right] \\
=\frac{\left(\gamma \ell_{P}^{2}\right)^{\frac{1}{2}}}{4^{\frac{1}{3}}} \sqrt[6]{|b c|}\left(\left(\frac{1}{2}-i \tau_{a}\right) \hat{e}_{a}^{+}+\left(\frac{1}{2}+i \tau_{a}\right) \hat{e}_{a}^{-}\right)\left[\left(\frac{1}{2}+i \tau_{a}\right)\right. \\
\left.\quad \times(\sqrt[6]{|a|}-\sqrt[6]{|a+1|})|\bar{a}+1\rangle+\left(\frac{1}{2}-i \tau_{a}\right)(\sqrt[6]{|a|}-\sqrt[6]{|a-1|})|\bar{a}-1\rangle\right] \\
=\frac{\left(\gamma \ell_{P}^{2}\right)^{\frac{1}{2}}}{4^{\frac{1}{3}}} \sqrt[6]{|b c|}\left[\left(\frac{1}{2}+i \tau_{a}\right)(\sqrt[6]{|a|}-\sqrt[6]{|a+1|})+\left(\frac{1}{2}-i \tau_{a}\right)(\sqrt[6]{|a|}-\sqrt[6]{|a-1|})\right]|\bar{a}\rangle,
\end{aligned}
$$

where $a, b$, and $c$ stand for one of $\mu_{v}, \nu_{v}$ or $k_{v}$ and $\bar{a}$ in the kets stands for $\mu_{v}, \nu_{v}$ or the pair $k_{ \pm}$. In this abbreviation for $|v\rangle$ the other labels are suppressed. When $\bar{a}=k_{ \pm}, 1$ is added or subtracted from both $k_{+}$and $k_{-}$. In the next two equations, where the vertex functions are eigenfunctions, we write simply $|v\rangle$. Hence,

$$
\begin{aligned}
\hat{h}_{a} & {\left[\hat{h}_{a}^{-1}, \hat{V}^{\frac{1}{3}}\right] \hat{h}_{b}\left[\hat{h}_{b}^{-1}, \hat{V}^{\frac{1}{3}}\right] \hat{h}_{c}\left[\hat{h}_{c}^{-1}, \hat{V}^{\frac{1}{3}}\right]|v\rangle=\frac{\left(\gamma \ell_{P}^{2}\right)^{\frac{3}{2}}}{4} \sqrt[3]{\left|k_{v}\right|\left|\mu_{v}\right|\left|\nu_{v}\right|} } \\
& \times\left[\left(\frac{1}{2}+i \tau_{3}\right)\left(\sqrt[6]{\left|k_{v}\right|}-\sqrt[6]{\left|k_{v}+1\right|}\right)+\left(\frac{1}{2}-i \tau_{3}\right)\left(\sqrt[6]{\left|k_{v}\right|}-\sqrt[6]{\left|k_{v}-1\right|}\right)\right] \\
& \times\left[\left(\frac{1}{2}+i \tau_{x}\right)\left(\sqrt[6]{\left|\mu_{v}\right|}-\sqrt[6]{\left|\mu_{v}+1\right|}\right)+\left(\frac{1}{2}-i \tau_{x}\right)\left(\sqrt[6]{\left|\mu_{v}\right|}-\sqrt[6]{\left|\mu_{v}-1\right|}\right)\right] \\
& \times\left[\left(\frac{1}{2}+i \tau_{y}\right)\left(\sqrt[6]{\left|\nu_{v}\right|}-\sqrt[6]{\left|\nu_{v}+1\right|}\right)+\left(\frac{1}{2}-i \tau_{y}\right)\left(\sqrt[6]{\left|\nu_{v}\right|}-\sqrt[6]{\left|\nu_{v}-1\right|}\right)\right]|v\rangle .
\end{aligned}
$$

After we take the trace, only the products including all $\tau$ terms or all non- $\tau$ terms will remain. That is

$$
\begin{aligned}
& \operatorname{Tr}\left[\hat{h}_{x}\left[\hat{h}_{x}^{-1}, \hat{V}^{\frac{1}{3}}\right] \hat{h}_{y}\left[\hat{h}_{y}^{-1}, \hat{V}^{\frac{1}{3}}\right] \hat{h}_{z}\left[\hat{h}_{z}^{-1}, \hat{V}^{\frac{1}{3}}\right]\right]|v\rangle=\frac{\left(\gamma \ell_{P}^{2}\right)^{\frac{3}{2}}}{4} \sqrt[3]{\left|k_{v}\right|\left|\mu_{v}\right|\left|\nu_{v}\right|} \\
& \times\left(\left[\sqrt[6]{\left|\mu_{v}\right|}-\frac{1}{2}\left(\sqrt[6]{\left|\mu_{v}-1\right|}+\sqrt[6]{\left|\mu_{v}+1\right|}\right)\right]\right. \\
& {\left[\sqrt[6]{\left|\nu_{v}\right|}-\frac{1}{2}\left(\sqrt[6]{\left|\nu_{v}-1\right|}+\sqrt[6]{\left|\nu_{v}+1\right|}\right)\right]\left[\sqrt[6]{\left|k_{v}\right|}-\frac{1}{2}\left(\sqrt[6]{\left|k_{v}-1\right|}+\sqrt[6]{\left|k_{v}+1\right|}\right)\right]} \\
& \left.+\frac{i}{8}\left(\sqrt[6]{\left|\mu_{v}-1\right|}-\sqrt[6]{\left|\mu_{v}+1\right|}\right)\left(\sqrt[6]{\left|\nu_{v}-1\right|}-\sqrt[6]{\left|\nu_{v}+1\right|}\right)\left(\sqrt[6]{\left|k_{v}-1\right|}-\sqrt[6]{\left|k_{v}+1\right|}\right)\right)|v\rangle
\end{aligned}
$$


so after summing over the Levi-Civita symbol we are left with

$$
\begin{aligned}
& \widehat{V^{-1}}\left|k_{ \pm}, \mu_{v}, \nu_{v}\right\rangle=-\frac{16 \hbar^{3} i}{81 \kappa^{3} \gamma^{3}} \frac{i\left(\gamma \ell_{P}^{2}\right)^{\frac{3}{2}}}{32} \sqrt[3]{\left|k_{v}\right|\left|\mu_{v}\right|\left|\nu_{v}\right|}\left(\sqrt[6]{\left|\mu_{v}-1\right|}-\sqrt[6]{\left|\mu_{v}+1\right|}\right) \\
& \left.\times\left(\sqrt[6]{\left|\nu_{v}-1\right|}-\sqrt[6]{\left|\nu_{v}+1\right|}\right)\left(\sqrt[6]{\left|k_{v}-1\right|}-\sqrt[6]{\left|k_{v}+1\right|}\right)\right)\left|k_{ \pm}, \mu_{v}, \nu_{v}\right\rangle \\
& =\frac{l_{p}^{3} \hbar^{3}}{162 \kappa^{3} \gamma^{\frac{3}{2}}} \sqrt[3]{\left|k_{v}\right|\left|\mu_{v}\right|\left|\nu_{v}\right|}\left(\sqrt[6]{\left|\mu_{v}-1\right|}-\sqrt[6]{\left|\mu_{v}+1\right|}\right)\left(\sqrt[6]{\left|\nu_{v}-1\right|}-\sqrt[6]{\left|\nu_{v}+1\right|}\right) \\
& \left.\times\left(\sqrt[6]{\left|k_{v}-1\right|}-\sqrt[6]{\left|k_{v}+1\right|}\right)\right)\left|k_{ \pm}, \mu_{v}, \nu_{v}\right\rangle
\end{aligned}
$$

which is the result in section IVC with $\mu_{0}=1=\nu_{0}$.

\section{Appendix B: Hamiltonian constraint quantization}

In $(3+1)$-LQG the kinetic part of the Hamiltonian constraint is regularized in terms of closed loops with a "tail." In the first term of the Hamiltonian constraint, denoted by $H_{K}^{1}$ (as usual, $H_{K}$ contributes with a minus sign to the full Hamiltonian constraint) the analogue

of closed loops are composed of point holonomies, the "tails" are sections of adjacent edges, see (B11) below.

Replacing the integral in the classical expression of the first part of the Hamiltonian constraint with test function $N$,

$$
H_{K}^{1}[N]=\frac{1}{\gamma^{2}} \int \mathrm{d} z N(z) \frac{X E^{x} Y E^{y}}{\sqrt{\mathcal{E} E^{x} E^{y}}}
$$

by a Riemann sum leads to terms

$$
\frac{1}{\gamma^{2}} \frac{\epsilon X(z) Y(z) E^{x}(z) E^{y}(z)}{V(z)}
$$

where we have made use of the volume expression $V=\sqrt{\mathcal{E} E^{x} E^{y}}$ and chosen $N \equiv 1$. For a representation by a Poisson bracket we take first the holonomy along an interval to the left of a vertex, $v$

$$
h_{z,-}=e^{\tau_{3} \int_{-} \mathcal{A}},
$$

where

$$
\int_{-}=\int_{z(v)}^{z(v)-\epsilon}=-\int_{z(v)-\epsilon}^{z(v)}=:-\int_{\mathcal{I}_{-}}
$$


in which $z(v)$ is the coordinate of a vertex. To express $H_{K}^{1}$ in terms of holonomies and fluxes, we first calculate the Poisson bracket

$$
\left\{h_{z,-}^{-1}, V\right\}=\kappa \gamma \int \mathrm{d} z^{\prime} \frac{\delta}{\delta \mathcal{A}\left(z^{\prime}\right)} e^{+\tau_{3} \int_{\mathcal{I}_{-}} \mathcal{A}(z) \mathrm{d} z} \frac{\delta}{\delta \mathcal{E}\left(z^{\prime}\right)} \epsilon \int \mathrm{d} z^{\prime \prime} V\left(z^{\prime \prime}\right) .
$$

The integration in the holonomy goes in the positive direction, so for every $z^{\prime} \in \mathcal{I}_{-}$the functional derivative with respect to $\mathcal{A}$ gives a factor

$$
\tau_{3} \delta\left(z-z^{\prime}\right)
$$

so that the integral over $z^{\prime}$ reduces to $\int_{\mathcal{I}_{-}} \mathrm{d} z^{\prime}$.

$$
\begin{aligned}
\left\{h_{z,-}^{-1}, V\right\} & \approx \kappa \gamma \tau_{3} e^{\tau_{3} \int_{\mathcal{I}_{-}} \mathcal{A}(z) \mathrm{d} z} \int_{\mathcal{I}_{-}} \mathrm{d} z^{\prime} \frac{\delta}{\delta \mathcal{E}\left(z^{\prime}\right)} \int \mathrm{d} z^{\prime \prime} \sqrt{\mathcal{E}\left(z^{\prime \prime}\right) E^{x}\left(z^{\prime \prime}\right) E^{y}\left(z^{\prime \prime}\right)} \\
& =\frac{\kappa \gamma}{2} \tau_{3} h_{z,-}^{-1} \int_{\mathcal{I}_{-}} \mathrm{d} z^{\prime} \frac{E^{x}\left(z^{\prime}\right) E^{y}\left(z^{\prime}\right)}{V\left(z^{\prime}\right)}
\end{aligned}
$$

Approximating the integral by the integrand at $z$ multiplied by the interval length $\epsilon$, we finally arrive at

$$
h_{z,-}\left\{h_{z,-}^{-1}, V\right\} \approx \frac{\kappa \gamma}{2} \tau_{3} \frac{\epsilon E^{x}(z) E^{y}(z)}{V(z)} .
$$

For the interval going to the right from the vertex, the integral $\int_{+} \mathcal{A}$ goes into the positive direction and

$$
\frac{\delta}{\delta \mathcal{A}} h_{z,+}^{-1}=-\tau_{3} h_{z,+}^{-1}
$$

In comparison with $(\underline{\mathrm{B} 6})$ this gives an overall minus sign. So both Poisson brackets give the desired classical approximation with different signs and we can symmetrize.

$$
\begin{aligned}
\frac{\kappa \gamma}{2} \tau_{3} \frac{\epsilon E^{x}(z) E^{y}(z)}{V(z)} & \approx \frac{1}{2}\left(h_{z,-}\left\{h_{z,-}^{-1}, V\right\}-h_{z,+}\left\{h_{z,+}^{-1}, V\right\}\right) \\
& =-\frac{1}{2} \sum_{\sigma} \sigma h_{z, \sigma}\left\{h_{z, \sigma}^{-1}, V\right\}
\end{aligned}
$$

where we have introduced the sign factor $\sigma= \pm 1$ of Ref. [10]. In analogy to full LQG this is multiplied by

$$
\begin{aligned}
& h_{x} h_{y} h_{x}^{-1} h_{y}^{-1}-h_{y} h_{x} h_{y}^{-1} h_{x}^{-1}=4 \tau_{x} \sin \left(\mu_{0} X\right) \sin ^{2}\left(\frac{\nu_{0}}{2} Y\right) \\
& -4 \tau_{y} \sin ^{2}\left(\frac{\mu_{0}}{2} X\right) \sin \left(\nu_{0} Y\right)+2 \tau_{3} \sin \left(\mu_{0} X\right) \sin \left(\nu_{0} Y\right)
\end{aligned}
$$

and the trace is taken. Because of the $\tau_{3}$ matrix in (B7), the only part of (B8) contributing to the trace is

$$
2 \tau_{3} \sin \left(\mu_{0} X\right) \sin \left(\nu_{0} Y\right)
$$


We find

$$
\begin{aligned}
& \operatorname{Tr}\left[\left\{h_{x} h_{y} h_{x}^{-1} h_{y}^{-1}-h_{y} h_{x} h_{y}^{-1} h_{x}^{-1}\right\}\left(-\frac{1}{2}\right) \sum_{\sigma} \sigma h_{z, \sigma}\left\{h_{z, \sigma}^{-1}, V\right\}\right] \\
& \approx \sum_{\sigma} \sigma \operatorname{Tr}\left[\sin \left(\mu_{0} X\right) \sin \left(\nu_{0} Y\right) \kappa \gamma \tau_{3}^{2} \frac{\epsilon E^{x} E^{y}}{V}\right] \\
& =-\frac{\kappa \gamma}{2} \frac{\epsilon E^{x}(z) E^{y}(z)}{V(z)} \sin \left(\mu_{0} X\right) \sin \left(\nu_{0} Y\right),
\end{aligned}
$$

and in first approximation of the sine function we have

$$
\begin{aligned}
& \sum_{\sigma} \sigma \operatorname{Tr}\left[\left\{h_{x} h_{y} h_{x}^{-1} h_{y}^{-1}-h_{y} h_{x} h_{y}^{-1} h_{x}^{-1}\right\} h_{z, \sigma}\left\{h_{z, \sigma}^{-1}, V\right\}\right] \\
& \approx \kappa \gamma \mu_{0} \nu_{0} \frac{\epsilon X(z) Y(z) E^{x}(z) E^{y}(z)}{V(z)} .
\end{aligned}
$$

Thus, when we replace the Poisson brackets by $(i \hbar)^{-1}$ times the commutator we obtain

$$
\hat{H}_{K}^{1}=\frac{1}{i \hbar \kappa \gamma^{3} \mu_{0} \nu_{0}} \sum_{\sigma} \sigma \operatorname{Tr}\left(\left\{\hat{h}_{x} \hat{h}_{y} \hat{h}_{x}^{-1} \hat{h}_{y}^{-1}-\hat{h}_{y} \hat{h}_{x} \hat{h}_{y}^{-1} \hat{h}_{x}^{-1}\right\} \hat{h}_{z, \sigma}\left[\hat{h}_{z, \sigma}^{-1}, \hat{V}\right]\right) .
$$

\section{Action on states}

Consider first the action of $\hat{h}_{z,-}\left[\hat{h}_{z,-}^{-1}, \hat{V}\right]$ on a vertex of a state $T_{G, \vec{k}, \vec{\mu}, \vec{\nu}}$ of the form (44), with the vertex function denoted shortly by $|v\rangle$, and all edge holonomies oriented from the left to the right.

$$
\begin{aligned}
\hat{h}_{z,-}^{-1} & =\cos \left(\frac{1}{2} \int_{-} \mathcal{A}\right)+2 \tau_{3} \sin \left(\frac{1}{2} \int_{-} \mathcal{A}\right) \\
& =\frac{1}{2}\left(1+2 i \tau_{3}\right) e^{\frac{i}{2} \int_{-} \mathcal{A}}+\frac{1}{2}\left(1-2 i \tau_{3}\right) e^{-\frac{i}{2} \int_{-} \mathcal{A}}
\end{aligned}
$$

As $\int_{-}$goes into the negative direction, $e^{\frac{i}{2} \int_{-} \mathcal{A}}=e^{-\frac{i}{2} \int_{\mathcal{I}_{-}} \mathcal{A}}$ lowers the label $k_{-}$of the edge $e^{-}$ (to the left of a vertex $v$ ) by one on the section $(z(v)-\epsilon, z(v)) \subset e^{-}$. For the same reason $e^{-\frac{i}{2} \int_{-} \mathcal{A}}$ raises the label $k_{-}$by one and so

$$
\begin{aligned}
\hat{V} \hat{h}_{z,-}^{-1}|v\rangle & =\frac{\gamma^{\frac{3}{2}} \ell_{\mathrm{P}}^{3}}{4}\left[\frac{1}{2}\left(1+2 i \tau_{3}\right) \sqrt{\left|\mu_{v}\right|\left|\nu_{v}\right|\left|k_{v}-1\right|} e^{\frac{i}{2} \int_{-} \mathcal{A}}\right. \\
& \left.+\frac{1}{2}\left(1-2 i \tau_{3}\right) \sqrt{\left|\mu_{v}\right|\left|\nu_{v}\right|\left|k_{v}+1\right|} e^{-\frac{i}{2} \int_{-} \mathcal{A}}\right]|v\rangle
\end{aligned}
$$

with the result that

$$
\begin{aligned}
\hat{h}_{z,-}\left[\hat{h}_{z,-}^{-1}, \hat{V}\right]|v\rangle= & \frac{\gamma^{\frac{3}{2}} \ell_{\mathrm{P}}^{3}}{4} \sqrt{\left|\mu_{v} \nu_{v}\right|} \times \\
& {\left[1-\frac{1}{2} \sqrt{\left|k_{v}+1\right|}-\frac{1}{2} \sqrt{\left|k_{v}-1\right|}\right.} \\
& \left.+i \tau_{3}\left(\sqrt{\left|k_{v}+1\right|}-\sqrt{\left|k_{v}-1\right|}\right)\right]|v\rangle .
\end{aligned}
$$


Inserting the parts containing $\tau_{3}$ of $(6)$ and $(\underline{B 14})$ into (B11) gives (including $\sigma=-1$ )

$$
\frac{\gamma^{\frac{3}{2}} \ell_{\mathrm{P}}^{3}}{4 \ell_{\mathrm{P}}^{2} \gamma^{3} \mu_{0} \nu_{0}} \sqrt{\left|\mu_{v}\right|\left|\nu_{v}\right|}\left(\sqrt{\left|k_{v}+1\right|}-\sqrt{\left|k_{v}-1\right|}\right) \sin \left(\mu_{0} X\right) \sin \left(\nu_{0} Y\right) .
$$

The analogous expression with $\hat{h}_{z,+}$ gives the same as (B14) with the opposite sign. With both signs of $\sigma$ we have the action on a gauge-invariant state (44) given in equation (72).

\section{Appendix C: Solution to the Hermitian Killing Constraint}

In this Appendix we work from equation (102) to derive solutions to the Hermitian Killing constraint, equation (104). For powers of $\hat{V}$ we have

$$
(\hat{V}[\mathcal{I}])^{q}\left|k_{ \pm}, \mu_{v}, \nu_{v}\right\rangle=\left(\frac{\gamma^{\frac{3}{2}} \ell_{P}^{3}}{4} \sqrt{\left|\mu_{v}\right|\left|\nu_{v}\right|\left|k_{v}\right|}\right)^{q}\left|k_{ \pm}, \mu_{v}, \nu_{v}\right\rangle
$$

and from equation (46) we may infer the action of $\operatorname{Tr}\left[\tau_{x} \hat{h}_{x}^{-1}[\mathcal{I}]\right]$ in which $\mathcal{I}$ is understood to contain the vertex $v$,

$$
\operatorname{Tr}\left[\tau_{x} \hat{h}_{x}^{-1}[\mathcal{I}]\right]\left|k_{ \pm}, \mu_{v}, \nu_{v}\right\rangle=i\left(\left|k_{ \pm}, \mu_{v}-\mu_{0}, \nu_{v}\right\rangle-\left|k_{ \pm}, \mu_{v}+\mu_{0}, \nu_{v}\right\rangle\right)
$$

with an analogous equation applying for $Y$. Combining these actions we have (writing $\mu \equiv \mu_{v}$ and $\left.\nu \equiv \nu_{v}\right)$

$$
\begin{aligned}
& \hat{\mathcal{K}}_{q}\left|k_{ \pm}, \mu, \nu\right\rangle=\frac{i\left(\gamma \ell_{P}^{2}\right)^{3 q+1}}{4^{2 q}}|k|^{q}\left(\frac { | \nu | ^ { q } } { \mu _ { 0 } } \left[\left|\mu\left(\mu-\mu_{0}\right)\right|^{\frac{q+1}{2}}\left|k_{ \pm}, \mu-\mu_{0}, \nu\right\rangle\right.\right. \\
&\left.-\left|\mu\left(\mu+\mu_{0}\right)\right|^{\frac{q+1}{2}}\left|k_{ \pm}, \mu+\mu_{0}, \nu\right\rangle\right] \\
&\left.+\frac{|\mu|^{q}}{\nu_{0}}\left[\left|\nu\left(\nu-\nu_{0}\right)\right|^{\frac{q+1}{2}}\left|k_{ \pm}, \mu, \nu-\nu_{0}\right\rangle-\left|\nu\left(\nu+\nu_{0}\right)\right|^{\frac{q+1}{2}}\left|k_{ \pm}, \mu, \nu+\nu_{0}\right\rangle\right]\right) .
\end{aligned}
$$

By direct calculation it is easy to see that

$$
\left\langle k_{ \pm}^{\prime}, \mu^{\prime}, \nu^{\prime}\left|\hat{\mathcal{K}}_{q}\right| k_{ \pm}, \mu, \nu\right\rangle=\left\langle k_{ \pm}, \mu, \nu\left|\hat{\mathcal{K}}_{q}\right| k_{ \pm}^{\prime}, \mu^{\prime}, \nu^{\prime}\right\rangle^{*}
$$

$\hat{\mathcal{K}}_{q}$ is a Hermitian operator.

With the superposition of vertex states of equation (103) we can use equation (C3) to establish the following recurrence relation involving the $a_{\mu, \nu}$ coefficients

$$
\begin{gathered}
0=\left|k_{v}\right|^{q}\left(\frac{|\nu|^{q}}{\mu_{0}}\left[\left|\mu\left(\mu+\mu_{0}\right)\right|^{\frac{q+1}{2}} a_{\mu+\mu_{0}, \nu}-\left|\mu\left(\mu-\mu_{0}\right)\right|^{\frac{q+1}{2}} a_{\mu-\mu_{0}, \nu}\right]\right. \\
\left.+\frac{|\mu|^{q}}{\nu_{0}}\left[\left|\nu\left(\nu+\nu_{0}\right)\right|^{\frac{q+1}{2}} a_{\mu, \nu+\nu_{0}}-\left|\nu\left(\nu-\nu_{0}\right)\right|^{\frac{q+1}{2}} a_{\mu, \nu-\nu_{0}}\right]\right) .
\end{gathered}
$$


Obviously, there exists a trivial solution, where $k_{v} \equiv k_{+}+k_{-}=0$. If we seek nontrivial solutions, we may safely assume $k_{+} \neq-k_{-}$and divide by $\left|k_{v}\right|^{q}$. Similarly, observe that in the case that either $\mu=0$ or $\nu=0$, the recurrence relation given in equation (C5) is again trivially satisfied [28], meaning if we seek additional solutions we may divide by $|\mu \nu|^{q}$

$$
\begin{aligned}
0 & =\frac{1}{\mu_{0}}\left(|\mu|^{\frac{1-q}{2}}\left|\mu+\mu_{0}\right|^{\frac{1+q}{2}} a_{\mu+\mu_{0}, \nu}-|\mu|^{\frac{1-q}{2}}\left|\mu-\mu_{0}\right|^{\frac{1+q}{2}} a_{\mu-\mu_{0}, \nu}\right) \\
& +\frac{1}{\nu_{0}}\left(|\nu|^{\frac{1-q}{2}}\left|\nu+\nu_{0}\right|^{\frac{1+q}{2}} a_{\mu, \nu+\nu_{0}}-|\nu|^{\frac{1-q}{2}}\left|\nu-\nu_{0}\right|^{\frac{1+q}{2}} a_{\mu, \nu-\nu_{0}}\right)
\end{aligned}
$$

The standard separation ansatz $a_{\mu, \nu}=\alpha_{\mu} \beta_{\nu}$ leads to

$$
-\mu_{0} C=|\mu|^{\frac{1-q}{2}}\left(\left|\mu+\mu_{0}\right|^{\frac{1+q}{2}} \alpha_{\mu+\mu_{0}}-\left|\mu-\mu_{0}\right|^{\frac{1+q}{2}} \alpha_{\mu-\mu_{0}}\right)
$$

and

$$
\nu_{0} C=|\nu|^{\frac{1-q}{2}}\left(\left|\nu+\nu_{0}\right|^{\frac{1+q}{2}} \beta_{\nu+\nu_{0}}-\left|\nu-\nu_{0}\right|^{\frac{1+q}{2}} \beta_{\nu-\nu_{0}}\right) .
$$

From the first of these relations we obtain

$$
\alpha_{\mu+\mu_{0}}=\left|\frac{\mu-\mu_{0}}{\mu+\mu_{0}}\right|^{\frac{q+1}{2}} \alpha_{\mu-\mu_{0}}-\frac{C \mu_{0}|\mu|^{\frac{q-1}{2}}}{\left|\mu+\mu_{0}\right|^{\frac{q+1}{2}}} .
$$

Iteration yields

$$
\alpha_{\mu+(2 m+1) \mu_{0}}=\left|\frac{\mu-\mu_{0}}{\mu+(2 m+1) \mu_{0}}\right|^{\frac{q+1}{2}} \alpha_{\mu-\mu_{0}}-\frac{\mu_{0} C}{\left|\mu+(2 m+1) \mu_{0}\right|^{\frac{q+1}{2}}} \sum_{l=0}^{m}\left|\mu+2 l \mu_{0}\right|^{\frac{q-1}{2}} .
$$

For large $m$ the finite sum can be approximated by

$$
\left(2 \mu_{0}\right)^{\frac{q-1}{2}} \sum_{l=0}^{m} l^{\frac{q-1}{2}}
$$

the leading term of which for $q \geq 1$ can be approximated by an integral

$$
\left(2 \mu_{0}\right)^{\frac{q-1}{2}} \frac{2 m^{\frac{q+1}{2}}}{q+1}
$$

So the second term in (C10) is, in leading order, equal to

$$
-\frac{C}{q+1}
$$

i.e. for large $m$ it goes to a constant as does $\alpha_{\mu+(2 m+1) \mu_{0}}$. As a consequence, for $C \neq 0$ we do not find nontrivial normalizable states, and we have to consider only the case $C=0$. 
With the reparametrizations $\mu \rightarrow \bar{\mu}+\mu_{0}$, where $\bar{\mu}$ is some initial value, and $m \rightarrow m-1$ we obtain from the first part of equation (C10)

$$
\alpha_{\bar{\mu}+2 m \mu_{0}}=\left|\frac{\bar{\mu}}{\bar{\mu}+2 m \mu_{0}}\right|^{\frac{q+1}{2}} \alpha_{\bar{\mu}} .
$$

For some given initial value $\alpha_{\bar{\mu}} \neq 0$ the series

$$
\sum_{m=0}^{\infty}\left|\alpha_{\bar{\mu}+2 m \mu_{0}}\right|^{2}
$$

converges, when $q>0$.

For a solution of the constraint equation we must also consider decreasing indices of $\alpha$. Analogous to equation (

$$
\alpha_{\bar{\mu}-2 m \mu_{0}}=\left|\frac{\bar{\mu}}{\bar{\mu}-2 m \mu_{0}}\right|^{\frac{q+1}{2}} \alpha_{\bar{\mu}} .
$$

The simplest nontrivial solution is obtained by choosing an initial value $\bar{\mu}=\mu_{0}$. Then from $m=1$ we find

$$
\alpha_{-\mu_{0}}=\alpha_{\mu_{0}}
$$

and

$$
\alpha_{ \pm(2 m+1) \mu_{0}}=\left|\frac{1}{2 m+1}\right|^{\frac{q+1}{2}}
$$

a symmetric series in $m$.

For even multiples of $\mu_{0}$ we find from equation (C11) that $\alpha_{2 \mu_{0}}=0$ (and $\alpha_{2 m \mu_{0}}=0$ for all $m$, irrespectively of the value of $\alpha_{0}$ ). On the other hand, if $\alpha_{-2 \mu_{0}} \neq 0, \alpha_{0}$ diverges. As a consequence, all states with even multiples of $\mu_{0}$ are excluded and we can also set $\alpha_{0}=0$, thus avoiding zero vacuum and zero length states, in accordance with the division by $|\mu \nu|^{q}$ in equation ( $(\mathrm{C} 6)$.

Under the assumption that $\bar{\mu}$ is an integer multiple of $\mu_{0}$, only the odd multiples are nonzero. In the following we make use of the abbreviation

$$
\alpha_{2 m+1} \equiv \alpha_{(2 m+1) \mu_{0}}
$$

For finite volume and length expectation values, and for finite second moments, both

$$
\sum_{m} \sqrt{|2 m+1|}\left|\alpha_{2 m+1}\right|^{2}
$$


and

$$
\sum_{m}|2 m+1|\left|\alpha_{2 m+1}\right|^{2}
$$

must converge. From the first condition follows

$$
q>\frac{1}{2}
$$

and from the second

$$
q>1 .
$$

So as with the commutator version, the Hermitian Killing operator $\hat{\mathcal{K}}$ must be volume weighted to give rise to finite length and volume expectation values and uncertainties.

To finish the solution we observe that the coefficients $\beta_{n}$ are found in the same way and starting with an initial value $\beta_{1}=1$ we have

$$
\beta_{ \pm(2 n+1)}=\left|\frac{1}{2 n+1}\right|^{\frac{q+1}{2}} .
$$

Combining these results we find coefficients $a_{2 m+1,2 n+1}=\alpha_{2 m+1} \beta_{2 n+1}$ for a solution with initial coefficient $a_{1,1}$

$$
a_{2 m+1,2 n+1}=\frac{a_{1,1}}{|(2 m+1)(2 n+1)|^{\frac{q+1}{2}}} .
$$

The norm square of this solution state to the Hermitian Killing constraint of equation (103), denoted by $|q\rangle$, is

$$
\langle q \mid q\rangle=\sum_{m, n=-\infty}^{\infty}\left|a_{2 m+1,2 n+1}\right|^{2}=4 a_{1,1}^{2} \sum_{m=0}^{\infty} \frac{1}{(2 m+1)^{q+1}} \sum_{n=0}^{\infty} \frac{1}{(2 n+1)^{q+1}},
$$

so that

$$
\||q\rangle \|=2 a_{1,1} \sum_{n=0}^{\infty} \frac{1}{(2 n+1)^{q+1}} .
$$

This sum can be written as

$$
\sum_{n=0}^{\infty} \frac{1}{(2 n+1)^{q+1}}+\sum_{n=1}^{\infty} \frac{1}{(2 n)^{q+1}}-\sum_{n=1}^{\infty} \frac{1}{(2 n)^{q+1}}=\sum_{n=1}^{\infty} \frac{1}{n^{q+1}}-\frac{1}{2^{q+1}} \sum_{n=1}^{\infty} \frac{1}{n^{q+1}}
$$

and expressed in terms of the Riemann zeta function. Thus,

$$
\||q\rangle \|=a_{1,1} \frac{2^{q+1}-1}{2^{q}} \zeta(q+1) .
$$

For

$$
a_{1,1}=\frac{2^{q}}{2^{q+1}-1} \frac{1}{\zeta(q+1)}
$$


we have a normalized state with finite expectation values

$$
|q\rangle=\frac{2^{q}}{2^{q+1}-1} \frac{1}{\zeta(q+1)} \sum_{m, n=-\infty}^{\infty} \frac{1}{|(2 m+1)(2 n+1)|^{\frac{q+1}{2}}}|2 m+1,2 n+1\rangle,
$$

where we have used the notation of equation (105). In the limit of large $q$ the state $|q\rangle$ goes to a superposition of four states with the same length and volume expectation values,

$$
\lim _{q \rightarrow \infty}|q\rangle \longrightarrow \frac{1}{2}(|1,1\rangle+|1,-1\rangle+|-1,1\rangle+|-1,-1\rangle) .
$$

Next we calculate the expectation value and the uncertainty of $\sqrt{|\mu \nu|}$ (up to a factor $\left.\sqrt{\mu_{0} \nu_{0}}\right)$, which is contained in both length and volume,

$$
W:=\langle\sqrt{|\mu \nu|}\rangle / \sqrt{\mu_{0} \nu_{0}}=4 \sum_{m, n=0}^{\infty} a_{2 m+1,2 n+1}^{2} \sqrt{(2 m+1)(2 n+1)},
$$

the factor 4 coming from the four quadrants in the $(m, n)$ plane. With the coefficients inserted from equation (C19)

$$
W=2\left(\frac{2^{q+\frac{1}{2}}-1}{2^{q+1}-1}\right)^{2}\left(\frac{\zeta\left(q+\frac{1}{2}\right)}{\zeta(q+1)}\right)^{2}
$$

In the same way we calculate

$$
\langle|\mu \nu|\rangle=4 \mu_{0} \nu_{0}\left(\frac{2^{q}-1}{2^{q+1}-1}\right)^{2}\left(\frac{\zeta(q)}{\zeta(q+1)}\right)^{2}
$$

and

$$
\Delta W=\left(\langle|\mu \nu|\rangle-\langle\sqrt{|\mu \nu|}\rangle^{2}\right)^{\frac{1}{2}} / \sqrt{\mu_{0} \nu_{0}}
$$

which gives equation (107),

$$
\Delta W=2 \frac{\left[\left(2^{q}-1\right)^{2}\left(2^{q+1}-1\right)^{2} \zeta(q)^{2} \zeta(q+1)^{2}-\left(2^{q+\frac{1}{2}}-1\right)^{4} \zeta\left(q+\frac{1}{2}\right)^{4}\right]^{\frac{1}{2}}}{\left(2^{q+1}-1\right)^{2} \zeta(q+1)^{2}} .
$$

In accordance with equation (106) the length and volume uncertainties go to zero for large $q$.

[1] C. Rovelli and L. Smolin Nucl. Phys. (1995) B 442 593; erratum Nucl. Phys. B 456 (1995) 753. 
[2] R. Loll "Spectrum of the Volume Operator in Quantum Gravity" Nucl. Phys. B460 (1996) 143-154 arXiv:gr-qc/9511030.

[3] A. Ashtekar and J. Lewandowski "Quantum Theory of Gravity I: Area Operators" Class. Quant. Grav. 14 (1997) A55-A82 arXiv:gr-qc/9602046; A. Ashtekar and J. Lewandowski "Quantum theory of geometry. II: Volume operators" Adv. Theor. Math. Phys. 1 (1998) 388 arXiv:gr-qc/9711031.

[4] T. Thiemann "A length operator for canonical quantum gravity" J. Math. Phys. 39 (1998) 3372-3392 arXiv:gr-qc/9606092

[5] S. Major, "Operators for quantized directions" Class. Quant. Grav. 16 (1999) 3859 arXiv:gr-qc/9905019.

[6] E. Bianchi "The length operator in Loop Quantum Gravity" Nucl. Phys. B 807 (2009) 591624, arXiv:0806.4710.

[7] F. Hinterleitner and S. Major, "On plane gravitational waves in real connection variables", Phys. Rev. D 83 044034, arXiv:1006.4146.

[8] F. Hinterleitner and S. Major, "Toward Loop Quantization of Plane Gravitational Waves" Class. Quantum Grav. 29 (2012) 065019, arXiv:1106.1448.

[9] K. Banerjee and G. Date "Loop Quantization of Polarized Gowdy Model on $T^{3}$ : Classical Theory", Class. Quantum Grav. 25 (2008) 105014, arXiv:0712.0683,

K. Banerjee and G. Date, "Loop Quantization of Polarized Gowdy Model on $T^{3}$ : Kinematical States and Constraint Operators" Class. Quantum Grav. 25 (2008) 145004, arXiv:0712.0687.

[10] M. Bojowald and R. Swiderski, "Spherically Symmetric Quantum Geometry: Hamiltonian Constraint," Class. Quantum Grav. 23 (2006) 2129-2154, arXiv:gr-qc/ 0511108.

[11] D. Neville, Class. Quantum Grav. 10 (1993) 2223; Phys. Rev. D 55 (1997) 766; Phys. Rev. D 55 (1997) 2069; Phys. Rev. D 56 (1997) 3485; Phys. Rev. D 57 (1998) 986.

[12] R. Borissov, Phys. Rev. D 49 (1994) 923.

[13] C. Beetle, "Midi-Superspace Quantization of Non-Compact Toroidally Symmetric Gravity" Adv. Theor. Math. Phys. 2 (1998) 471-495 arXiv:gr-qc/9801107.

[14] G. A. Mena Marugan and M. Montejo, "Quantization of pure gravitational plane waves" Phys. Rev. D 58 (1998) 104017 arXiv:gr-qc/9806105.

[15] T. Thiemann, "Quantum Spin Dynamics (QSD)", Class. Quantum Grav. 15 (1998) 139-173, arXiv:gr-qc/960608. 
[16] T. Thiemann and O. Winkler, "Gauge Field Theory Coherent States (GCS) : IV. Infinite Tensor Product and Thermodynamical Limit", Class. Quantum Grav. 18 (2001) 4997-5054, arXiv:hep-th/0005235

[17] J. B. Griffiths and J. Podolský 2009 Exact Space-Times in General Relativity (Cambridge: Cambridge University Press).

[18] M. Bojowald, "Isotropic Loop Qauntum Cosmology", Class. Quantum Grav. 19 (2002) 27172742, arXiv:gr-qc/0202077,

M. Bojowald, "Loop Quantum Cosmology", Living Rev. Relativity, 11 (2008) 4, http://www.livingreviews.org/lrr-2008-4.

[19] T. Thiemann, Modern Canonical Quantum General Relativity (Cambridge, 2007).

[20] C. Misner, K. Thorne, J. Wheeler, Gravitation (W. H. Freeman and Company, New York, 1973), section 35.9 .

[21] F. Scardigli, "Generalized Uncertainty Principle in Quantum Gravity from Micro-Black Hole Gedanken Experiment" Phys. Lett. B452 (1999) 39, arXiv:hep-th/9904025.

[22] S. Hossenfelder, "Minimal Length Scale Scenarios for Quantum Gravity" Living Rev. Relativity 16 (2013) 2, arXiv:1203.6191.

[23] B. Carr, L. Modesto and I. Prémont-Schwarz, "Generalized Uncertainty Principle and Selfdual Black Holes", arXiv:1107.0708.

[24] In Ref. 7] we used $\kappa^{\prime}=4 \pi G / A_{o}$ for the fiducial area in the transverse plane, but the form of this constant will play no role in the present work.

[25] This is not quite trivial, because in quantum theory the inverse volume in these expressions, when promoted to an operator, contains holonomies and so does not commute with $G$. But, according to the usual factor ordering prescription [19], holonomies stand left of triads, so that solutions of the Gauß constraint are indeed annihilated by these parts of $H$.

[26] The details of the calculation are carried out in the same way as in the first part of the Hamiltonian constraint, with $\mathcal{I}$ partitioned into a part left and a part right of the vertex, see below. When $\mathcal{I}$ is taken as a whole, the expression in parentheses in (58) is replaced by $\frac{1}{2}\left(\sqrt{\left|k_{v}+2\right|}-\sqrt{\left|k_{v}-2\right|}\right)$.

[27] More precisely, the operator $\mathcal{K}_{-1 / 2}=p_{\ell} / \mathcal{E}$. We have neglected the contribution of $\mathcal{E}$ due to the first Killing constraint and the constancy of $k_{v}$.

[28] Provided $q \neq 1$. However if this is the case, then setting $\mu=0$ and $\nu \neq 0$ (for instance) reduces 
eq. (C5) to $a_{\mu+\mu_{0}, \nu}=a_{\mu-\mu_{0}, \nu}$, which clearly admits no normalizable, nondegenerate solutions. 